\title{
Quasi-periodic solutions for a Schrödinger equation with a quintic nonlinear term depending on the time and space variables
}

\section{Yi Wang ${ }^{1 *}$, Jie Liu ${ }^{2}$ and Min Zhang ${ }^{3}$}

\section{"Correspondence:}

yiwang8080@126.com

${ }^{1}$ School of Mathematics and

Quantitative Economics, Shandong

University of Finance and

Economics, Jinan, P.R. China

Full list of author information is

available at the end of the article

\begin{abstract}
This article is devoted to the study of a nonlinear Schrödinger equation with an $x$-periodic and $t$-quasi-periodic quintic nonlinear term. It is proved that the equation admits small-amplitude, linearly stable, real analytic, and quasi-periodic solutions for most values of frequency vector. By utilizing the measure estimation of infinitely many small divisors, we construct a real analytic, symplectic change of coordinates which can transform the Hamiltonian into some sixth order Birkhoff normal form. We show an infinite-dimensional KAM theorem for non-autonomous Schrödinger equations and apply the theorem to prove the existence of quasi-periodic solutions.
\end{abstract}

Keywords: Schrödinger equation; Quintic nonlinear term; Quasi-periodically forced; $x$-dependent; Quasi-periodic solution; KAM theory

\section{Introduction}

In this paper, a Schrödinger equation with an $x$-periodic and $t$-quasi-periodic quintic nonlinear term

$$
\mathrm{i} u_{t}-u_{x x}+\tilde{m} u+\varepsilon g(\omega t, x)|u|^{4} u=0
$$

under the Dirichlet boundary conditions

$$
u(t, 0)=u(t, \pi)=0
$$

is considered, where $\tilde{m}$ is real; $\varepsilon$ is a small positive parameter; $\omega \in[\varrho, 2 \varrho]^{\kappa}(\varrho>0)$ is a $\kappa$-dimensional frequency vector; $\kappa \geq 1$ is an integer; and the function $g(\omega t, x)=g(\vartheta, x)$, $(\vartheta, x) \in \mathbb{T}^{\kappa} \times[0, \pi]$ is real analytic in $(\vartheta, x)$ and quasi-periodic in $t$. We aim to explore whether the boundary value problem (1) with (2) has real analytic, linearly stable, and quasi-periodic solutions.

We study this equation as an infinite-dimensional Hamiltonian system. There are two main approaches to construct the periodic and quasi-periodic solutions. One is the CraigWayne-Bourgain (CWB) method and the other is the infinite-dimensional KAM theory. The KAM method can capture more properties of quasi-periodic solutions such as their

(c) The Author(s) 2018. This article is distributed under the terms of the Creative Commons Attribution 4.0 International License (http://creativecommons.org/licenses/by/4.0/), which permits unrestricted use, distribution, and reproduction in any medium, provided you give appropriate credit to the original author(s) and the source, provide a link to the Creative Commons license, and indicate if changes were made. 
Whitney smooth dependence on parameters, their Floquet forms, zero Lyapunov exponents, and linear stability. The KAM theory for partial differential equations was originated by Kuksin [1-3] and Wayne [4]. In this paper, we apply the KAM theory as well as Birkhoff normal forms to attain real analytic quasi-periodic solutions.

Kuksin and Pöschel [5] developed the KAM method to study the existence of small amplitude quasi-periodic solutions corresponding to any finite number of Fourier modes for perturbations of 1D nonlinear Schrödinger equation

$$
\mathrm{i} u_{t}-u_{x x}+m u+|u|^{2} u=0, \quad m>0,
$$

with the Dirichlet boundary condition. Geng and You [6] obtained a KAM theorem for (3) with periodic boundary conditions. One year later, they [7] gave a KAM theorem, which can be applied to the higher-dimensional Schrödinger equations with nonlocal smooth nonlinearity

$$
\mathrm{i} u_{t}-\Delta u+M_{\xi} u+f\left(|u|^{2}\right) u=0, \quad x \in \mathbb{R}^{d},
$$

where $M_{\xi}$ is a Fourier multiplier, $f$ is real analytic and vanishing at zero. Liu and Yuan [8] showed that the derivative nonlinear Schrödinger equation

$$
\mathrm{i} u_{t}+u_{x x}+\mathrm{i}\left(|u|^{2} u\right)_{x}=0
$$

possesses Cantor families of smooth quasi-periodic solutions of small amplitude. We note that the 1D nonlinear Schrödinger equation (3) is completely integrable, hence one can perturb any finite number of Fourier modes to obtain small amplitude quasi-periodic solutions, which is however not the case if the nonlinearities are of high order.

For Schrödinger equations with high order nonlinearities, Liang and You [9] obtained quasi-periodic solutions corresponding to any finite number of admissible Fourier modes for the Schrödinger equation

$$
\mathrm{i} u_{t}-u_{x x}+m u+|u|^{4} u=0, \quad m \in \mathbb{R}
$$

with Dirichlet boundary conditions. Geng and Yi [10] proved that (4) with the periodic boundary condition admits a Whitney smooth family of quasi-periodic solutions. Liang [11] considered the Schrödinger equation

$$
\mathrm{i} u_{t}-u_{x x}+|u|^{2 p} u=0, \quad p \in \mathbb{N}
$$

and proved the existence of quasi-periodic solutions corresponding to two-dimensional invariant tori. Geng and $\mathrm{Wu}$ [12] showed that the one-dimensional derivative nonlinear Schrödinger equation

$$
\mathrm{i} u_{t}-u_{x x}-\mathrm{i}\left(|u|^{4} u\right)_{x}=0
$$

admits a Whitney smooth family of real analytic quasi-periodic solutions with two Diophantine frequencies. Gao and Liu [13] proved that there are many two-dimensional el- 
liptic invariant tori, and thus quasi-periodic solutions for the nonlinear wave equation

$$
u_{t t}-u_{x x}+m u+u^{5}=0
$$

with Dirichlet boundary conditions. Comparing these equations with cubic nonlinearities, it is more difficult to find the quasi-periodic solutions for the equations with high order nonlinearities.

However, the models in [10-13] cannot explicitly contain the space variable. The authors in [10-12] applied the compact form condition, the generalized compact form condition, or the gauge invariant property. Although these conditions simplify the normal forms and measure estimates, the equations they [10-12] considered cannot explicitly contain the space variable $x$. The $x$-dependent nonlinearity implies that the equation is variant in space translations and the momentum is not conserved. After one expands the perturbation by infinite-dimensional coordinates, the coefficients are integrals of eigenfunctions' products. For the $x$-independent nonlinearity, infinite coefficients are zeros, and one only needs to prove that essentially finite divisors are not zero. For instance, in [10], the coefficients with $k_{1} n_{1}+k_{2} n_{2}+\sum_{n}\left(\alpha_{n}-\beta_{n}\right) n \neq 0$ are zero, and the authors can only discuss the divisors with $k_{1} n_{1}+k_{2} n_{2}+\sum_{n}\left(\alpha_{n}-\beta_{n}\right) n=0$. In multi-dimensional equations, the $x$-dependent nonlinearity has the effect that the normal form is not diagonal in the purely elliptic directions.

Eliasson and Kuksin [14] considered the $d$-dimensional nonlinear Schrödinger equation

$$
-\mathrm{i} \dot{u}=\Delta u+V(x) * u+\varepsilon \frac{\partial F(x, u, \bar{u})}{\partial \bar{u}}
$$

where $V(x)=\sum \hat{V}(a) e^{\mathrm{i}|a, x\rangle}$ is an analytic function and $F$ is real analytic. They proved that the solution $u$ persists as a quasi-periodic solution which has all Lyapunov exponents equal to zero and whose linearized equation is reducible to constant coefficients. This potential term is a convolution, and the nonlinear term cannot depend on $t$. Bambusi et al. [15] dealt with a degenerate KAM theory for lower-dimensional elliptic tori of infinite-dimensional Hamiltonian systems and applied it to the wave equation

$$
u_{t t}-u_{x x}+V(x) u+\xi u+f(x, u)=0
$$

They used the mass $\xi \in \mathbb{R}$ as a parameter. The result cannot be used to (1), since $g(\omega t, x)$ has $\kappa$ parameters. Eliasson et al. [16] thought the case with constant-coefficient nonlinearity $g(x, u)=g(u)$ is significantly easier than the general case. They [16] proved a KAM result for the nonlinear beam equation on the $d$-dimensional torus

$$
u_{t t}+\Delta^{2} u+m u+g(x, u)=0, \quad t \in \mathbb{R}, x \in \mathbb{T}^{d}
$$

where $g(x, u)=4 u^{3}+O\left(u^{4}\right)$. They showed that, for generic $m>0$, most of the small amplitude invariant finite dimensional tori of the linear equation persist as invariant tori of the nonlinear equation. However, the nonlinearity $u^{3}$ in (5) is still constant-coefficient and cannot depend on $x$. Jiao and Wang [17] constructed quasi-periodic solutions for a quasiperiodically forced and $x$-dependent one-dimensional Schrödinger equation. Their frequency vector is fixed, the boundary condition is parameterized, and the nonlinear term 
is cubic. Berti and Bolle [18] presented existence results of $C^{\infty}$ quasi-periodic solutions for Schrödinger equations with a multiplicative potential. Their proofs are based on an improved Nash-Moser iterative scheme and a multi-scale inductive analysis for the inverse linearized operators. Berti et al. [19] introduced a weighted majorant norm of vector field to handle the problem about the $x$ variable. In this paper, we only assume $g$ is bounded, i.e., $\left(\mathbf{H}_{2}\right)$. The decay property plays an important role, e.g., (51).

For an equation with a constant-coefficient nonlinearity, a constant-coefficient symplectic transformation can change one Hamiltonian to another; while for an equation with a variable-coefficient nonlinearity, a constant-coefficient transformation is not sufficient. For (1), we construct a quasi-periodic symplectic transformation. For quasi-periodically forced equation, see [20, 21].

Equation (1) is quasi-periodically forced, and has an $x$-dependent quintic nonlinearity. This equation simultaneously has three characters, which makes the problem more intricate and difficult. To the best of our knowledge, there are no results regarding the quasi-periodic solutions for (1) by the KAM method.

Observing that (1) has a quintic term, we would like to eliminate the non-normal form terms and get a sixth order Birkhoff normal form. Our purpose is that the remained terms after normal form procedure must have the form of $\left|q_{i}\right|^{2}\left|q_{j}\right|^{2}\left|q_{k}\right|^{2}$ or $\left|q_{i}\right|^{2}\left|q_{j}\right|^{2}\left|w_{k}\right|^{2}$. To obtain the normal form, we construct a symplectic transformation. But the coefficients of this transformation have divisors. Thus, we choose the indices (the admissible index) to control the divisors not equal to zero. Since the nonlinearity of (1) quasi-periodically depends on $t$, the transformation should be quasi-periodic. To deal with the variable $x$, motivated by [17], we divide the perturbation to the mean value part and the non-mean value part. With the help of the admissible index, the term with respect to the mean value, i.e., $\frac{1}{6} \sum_{i, j, d, l, m, n} G_{i j d l m n} q_{i} q_{j} q_{d} \bar{q}_{l} \bar{q}_{m} \bar{q}_{n}$, can be changed to normal form easily. However, the left term $\frac{1}{6} \sum_{|k| \geq 1, i, j, d, l, m, n} G_{k, i j d l m n} e^{\mathrm{i}|k, \vartheta\rangle} q_{i} q_{j} q_{d} \bar{q}_{l} \bar{q}_{m} \bar{q}_{n}$ can not. To resolve this problem, we add the small divisor condition

$$
\left|\langle k, \omega\rangle+\lambda_{i}+\lambda_{j}+\lambda_{d}-\lambda_{l}-\lambda_{m}-\lambda_{n}\right| \geq \frac{\varrho \varepsilon^{\eta_{0}}}{|k|^{\kappa+\eta_{1}}} .
$$

Obviously, the parameters set satisfying the condition should not be empty, which can be indicated by estimating their measure bigger than zero. The estimate is a complex and meticulous job. But the difficulty is more than that. The analyticity and regularity of transformation are difficult to be proved in the space $l^{a, s}$. To this end, we use the Fourier cosine expansion and construct an inequality-Lemma 3.3. After we obtain the sixth order normal form, we scale this normal form and establish a KAM theorem to find out the quasi-periodic solution.

If parameters satisfy the small divisor conditions, we can retain them in the original parameter set $\Pi$. If parameters do not satisfy these conditions, we throw them away. Generally, if $\Pi$ is not big, such as $[0,1]^{\kappa+b}$, then the measure of thrown parameters set is almost zero and the left "good" parameters set $\Pi_{\epsilon}$ possesses a positive measure. But if we choose $\Pi=[0,1]^{\kappa+b}$ in this paper, then $\Pi_{\epsilon}$ is too small and has a negative measure. So we select $\Pi=\left[\varepsilon^{-\frac{1}{12}}, 2 \varepsilon^{-\frac{1}{12}}\right]^{\kappa+b}$. There are two purposes. One is to obtain $\Pi_{\epsilon}$ with positive measure. The other is to ensure the smallness of vector field $\left|X_{P}\right|_{r}^{*}$. Although the thrown parameters set is big, the $\Pi$ is also big and the "good" parameters set $\Pi_{\epsilon}$ still has a positive measure. 
Unfortunately, since the measure of $\Pi$ reduces as $k+b$ reduces, we only can find quasiperiodic solutions for $\kappa+b>12$.

The paper is organized as follows. In Sect. 2, the main result, remarks, and comments are given, and we transform the equation to an infinite-dimensional Hamiltonian system. Section 3 is devoted to a sixth order Birkhoff normal form. In Sect. 4, we show an infinitedimensional KAM theorem and the measure estimates. Using this theorem, we prove our main result. Conclusions are made in Sect. 5. A few of lemmas are proved in the last section (Appendix).

\section{The Hamiltonian setting}

When $\varepsilon=0$, equation (1) becomes

$$
\mathrm{i} u_{t}-u_{x x}+\tilde{m} u=0 .
$$

Let $\phi_{j}(x)=\sqrt{\frac{2}{\pi}} \sin j x$ and $\lambda_{j}=j^{2}+\tilde{m}, j \in \mathbb{Z}^{+}:=\{1,2, \ldots\}$ be the basic modes and their frequencies for the linear Schrödinger equation (6) with Dirichlet boundary conditions, respectively. Every solution of (6) can be written as a super-position of the basic modes, namely

$$
u(t, x)=\sum_{j \geq 1} q_{j}(t) \phi_{j}(x), \quad q_{j}(t)=q_{j}^{0} e^{i \lambda_{j} t}
$$

Concerning the existence of quasi-periodic solutions for the nonlinear Schrödinger equation (1) with (2), we prove the following Theorem 2.1 which is the main result of this paper. Before that, we first make some assumptions. Define $D_{1}\left(\sigma_{1}\right):=\left\{\vartheta|| \operatorname{Im} \vartheta \mid<\sigma_{1}\right\}$, $D_{2}(2 a):=\{x|| \operatorname{Im} x \mid<2 a\}$,

$$
|g|_{\sigma_{1}, 2 a}:=\sup _{(\vartheta, x) \in D_{1}\left(\sigma_{1}\right) \times D_{2}(2 a)}|g(\vartheta, x)|,
$$

and $|g|_{2 a}:=\sup _{x \in D_{2}(2 a)}|g(\vartheta, x)|$ for every fixed $\vartheta \in D_{1}\left(\sigma_{1}\right)$. We assume $\tilde{\sigma}_{1}$ to be a fixed small positive number. Throughout this paper, we suppose that:

$\left(\mathbf{H}_{1}\right) g(\vartheta, x)=g_{0}+\sum_{k \in \mathbb{Z}^{\kappa} \backslash\{0\}} g_{k}(x) e^{\mathrm{i}\langle k, \vartheta\rangle}, 0 \neq g_{0} \in \mathbb{R}$, where $\langle\cdot, \cdot\rangle$ denotes the standard inner product in $\mathbb{C}^{\kappa}$.

$\left(\mathbf{H}_{2}\right)$ For some $\sigma_{1}>\tilde{\sigma}_{1}$ and $a>0, g$ analytically in $\vartheta, x$ extends to the domain $D_{1}\left(\sigma_{1}\right) \times$ $D_{2}(2 a)$ and $g$ is bounded in $D_{1}\left(\sigma_{1}\right) \times D_{2}(2 a)$ with finite norm $|g|_{\sigma_{1}, 2 a}$.

$\left(\mathbf{H}_{3}\right) \partial_{x}^{2 k+1} g(\vartheta, 0)=0, \forall k \in \mathbb{N}$.

Theorem 2.1 Let $b \geq 2$ be an integer with $\kappa+b>12$. Assume $\left(\mathbf{H}_{1}\right),\left(\mathbf{H}_{2}\right)$, and $\left(\mathbf{H}_{3}\right)$ hold. For each admissible index set $\mathcal{I}=\left\{n_{1}<n_{2}<\cdots<n_{b}\right\} \subset \mathbb{Z}^{+}$, there is a constant $\varepsilon^{*}$ such that, for any $0<\varepsilon<\varepsilon^{*}$, there exist a subset $\Omega \subset[\varrho, 2 \varrho]^{\kappa}$ with meas $\Omega>0$ and a subset $\Pi_{\varepsilon} \subset \Omega \times[0,1]^{b}$ with meas $\Pi_{\varepsilon}>0$ which satisfy that for any $\xi=\left(\omega, \varsigma_{n_{1}}, \ldots, \varsigma_{n_{b}}\right) \in \Pi_{\varepsilon}$, the boundary value problem (1) with (2) has a quasi-periodic solution.

Remark 2.1 Equation (1) has a large Cantor family of rotational $\kappa+b$-dimensional tori with frequency vector $\hat{\omega}_{*}$, where $\hat{\omega}_{*}=\left(\omega_{* 1}, \omega_{* 2}, \ldots, \omega_{* \kappa}, \varpi_{* n_{1}}, \varpi_{* n_{2}}, \ldots, \varpi_{* n_{b}}\right)$, with $\omega_{* i}=$ $\omega_{i}+\mathcal{O}(\varepsilon), 1 \leq i \leq \kappa$, and $\varpi_{* n_{j}}=\lambda_{n_{j}}+\mathcal{O}(\varepsilon), 1 \leq j \leq b$. 
Remark 2.2 In $\left(\mathbf{H}_{2}\right), g$ is defined in the strip $D_{2}(2 a)$. In fact, $g$ can be defined in any strip $D_{2}(\hat{a})$ as long as $\hat{a}>a$. The fixed positive $a$ will be used to define the Hilbert space $l^{a, s}$ below.

Remark 2.3 The definition (Definition 3.1) of admissible index sets is given in Sect. 3. There exists an infinite admissible index set $\mathcal{I}$ (see Sect. 3).

Remark 2.4 We use "meas" to represent the Lebesgue measure.

Remark 2.5 In this paper, $C$ denotes a universal constant if we do not care about its value.

Remark 2.6 Using the method of this paper, we cannot expect an existence result of quasiperiodic solutions $u(t, x)$ with the same frequency vector $\omega$ as $g$. That is because we add extra parameters while we use the KAM method. Extra parameters cause solutions with additional frequencies.

Remark 2.7 Actually, $g$ may be identified with analytic functions which are even on $x \in \mathbb{R}$, periodic in $x$, and quasi-periodic in $t$. However, equation (1) is defined on $x \in[0, \pi]$. So we put forward the hypothesis $\left(\mathbf{H}_{3}\right)$ so that $g(\omega t, \cdot)$ can be even expanded on $\mathbb{R}$ analytically.

We study equation (1) with (2) as the Hamiltonian system

$$
\dot{u}=\mathrm{i} \nabla H(u), \quad H=\frac{1}{2}(A u, u)+\frac{\varepsilon}{6} \int_{0}^{\pi} g(\omega t, x)|u|^{6} d x,
$$

where $A=-\frac{d^{2}}{d x^{2}}+\tilde{m}$, the inner product $(u, v)=\operatorname{Re} \int_{0}^{\pi} u \bar{v} d x$, and the gradient of $H$ is defined with respect to $(\cdot, \cdot)$.

Introduce the coordinates $q=\left(q_{1}, q_{2}, \ldots\right)$ through the ansatz

$$
u(t, x)=\sum_{j \geq 1} q_{j}(t) \phi_{j}(x)
$$

The coordinates are taken from the Hilbert space $l^{a, s}\left(a>0, s>\frac{1}{2}\right)$ of all complex valued infinite sequences

$$
l^{a, s}=l^{a, s}(\mathbb{C}):=\left\{z=\left(z_{1}, z_{2}, \ldots\right), z_{i} \in \mathbb{C}, i \geq 1 \text { s.t. }\left(\|z\|_{a, s}\right)^{2}=\sum_{i \geq 1}\left|z_{i}\right|^{2} i^{2 s} e^{2 a i}<\infty\right\} .
$$

Thus, we obtain the Hamiltonian

$$
H=\Lambda+\varepsilon G
$$

where

$$
\Lambda=\frac{1}{2} \sum_{j \geq 1} \lambda_{j}\left|q_{j}\right|^{2} \quad \text { and } \quad G=\frac{1}{6} \int_{0}^{\pi} g(\omega t, x)\left|\sum_{j \geq 1} q_{j}(t) \phi_{j}(x)\right|^{6} d x \text {. }
$$


The corresponding equations of motion are

$$
\dot{q}_{j}=2 \mathrm{i} \frac{\partial H}{\partial \bar{q}_{j}}, \quad j \geq 1,
$$

with respect to the symplectic structure $\frac{i}{2} \sum d q_{j} \wedge d \bar{q}_{j}$ on $l^{a, s}$.

Lemma 2.1 Let $I \subseteq \mathbb{R}$ be an interval and a curve $I \rightarrow l^{a, s}, t \rightarrow q(t)$ be an analytic solution of (8), then

$$
u(t, x)=\sum_{j \geq 1} q_{j}(t) \phi_{j}(x)
$$

is a classical solution of (1) that is analytic on $I \times[0, \pi]$.

From the above lemma, $u$ is a classical solution of (1). So we need to find a solution having the form (9). The proof is common and we omit it. Details can be found in [5].

Introducing a pair of action-angle variables $(J, \vartheta) \in \mathbb{R}^{\kappa} \times \mathbb{T}^{\kappa}\left(\mathbb{T}^{\kappa}:=\mathbb{R}^{\kappa} / 2 \pi \mathbb{Z}^{\kappa}\right)$, one can obtain an equivalent Hamiltonian that does not depend on the time variable. The autonomous formulation of our problem is reached as follows:

$$
\dot{q}_{j}=2 \mathrm{i} \frac{\partial H}{\partial \bar{q}_{j}}, \quad j \geq 1, \quad \dot{\vartheta}=\omega, \quad \dot{J}=-\varepsilon \frac{\partial G}{\partial \vartheta}=-\varepsilon \frac{\partial \int_{0}^{\pi} \chi d x}{\partial \vartheta},
$$

which is a Hamiltonian system with the Hamiltonian

$$
H=\langle\omega, J\rangle+\frac{1}{2} \sum_{j \geq 1} \lambda_{j}\left|q_{j}\right|^{2}+\varepsilon G(q, \vartheta)
$$

and the symplectic structure $d \vartheta \wedge d J+\frac{\mathrm{i}}{2} \sum d q_{i} \wedge d \bar{q}_{i}$, where $\langle\cdot, \cdot\rangle$ is the standard inner product in $\mathbb{C}^{\kappa}$.

To continue our investigation for Hamiltonian (10), we need to establish the regularity of the nonlinear Hamiltonian vector field $X_{G}$ associated to $G$.

Let $l_{b}^{2}$ and $L^{2}$, respectively, be the Hilbert spaces of all bi-infinite, square summated sequences with complex coefficients and all square-integrable complex-valued functions on $[-\pi, \pi]$. Suppose that

$$
\mathcal{F}: l_{b}^{2} \rightarrow L^{2}, \quad q \mapsto \mathcal{F} q=\frac{1}{\sqrt{2 \pi}} \sum_{j} q_{j} e^{\mathrm{i} j x}
$$

is the inverse discrete Fourier transform, which defines an isometry between the two spaces. The subspaces $l_{b}^{a, s} \subset l_{b}^{2}$ consist, by definition, of all bi-infinite sequences with the finite form

$$
\left(\|q\|_{a, s}\right)^{2}=\left|q_{0}\right|^{2}+\sum_{i}\left|q_{i}\right|^{2}|i|^{2 s} e^{2 a|i|}
$$

Through $\mathcal{F}$ they define subspaces $W^{a, s}[-\pi, \pi] \subset L^{2}[-\pi, \pi]$ that are normed by setting $\|\mathcal{F} q\|_{a, s}=\|q\|_{a, s}$. The following Lemma 2.2 has been proven in [5]. Using it, we can prove a regularity result-Lemma 2.3 . 
Lemma 2.2 For $a>0$ and $s>\frac{1}{2}$, the space $l_{b}^{a, s}$ is a Hilbert algebra with respect to convolution of the sequences $(q * p)_{j}:=\sum_{k} q_{j-k} p_{k}$, and

$$
\|q * p\|_{a, s} \leq c\|q\|_{a, s}\|p\|_{a, s}
$$

where the constant c depends only on s. Consequently, $W^{a, s}$ is a Hilbert algebra with respect to multiplication of functions.

Lemma 2.3 The Hamiltonian vector-field $X_{G}$ is real analytic as a map from some neighborhood of origin in $l^{a, s}$ into $l^{a, s}$, with $\left\|G_{\bar{q}}\right\|_{a, s}=\mathcal{O}\left(\left(\|q\|_{a, s}\right)^{5}\right)$.

See the proof in Appendix.

\section{Partial Birkhoff normal form}

Since $u=\sum_{j \geq 1} q_{j}(t) \phi_{j}(x)$, we attain that

$$
G(q, \vartheta)=\frac{1}{6} \sum_{i, j, d, l, m, n} \int_{0}^{\pi} g(\vartheta, x) \phi_{i} \phi_{j} \phi_{d} \phi_{l} \phi_{m} \phi_{n} d x q_{i} q_{j} q_{d} \bar{q}_{l} \bar{q}_{m} \bar{q}_{n} .
$$

From $\left(\mathbf{H}_{1}\right)$,

$$
\begin{aligned}
G(q, \vartheta)= & \frac{1}{6} \sum_{i, j, d, l, m, n} G_{i j d l m n} q_{i} q_{j} q_{d} \bar{q}_{l} \bar{q}_{m} \bar{q}_{n} \\
& +\frac{1}{6} \sum_{|k| \geq 1, i j, d, l, m, n} G_{k, i j d l m n} e^{\mathrm{i}\langle(k, \vartheta)} q_{i} q_{j} q_{d} \bar{q}_{l} \bar{q}_{m} \bar{q}_{n},
\end{aligned}
$$

where

$$
G_{i j d l m n}=g_{0} \int_{0}^{\pi} \phi_{i} \phi_{j} \phi_{d} \phi_{l} \phi_{m} \phi_{n} d x
$$

and

$$
G_{k, i j d l m n}=\int_{0}^{\pi} g_{k}(x) \phi_{i} \phi_{j} \phi_{d} \phi_{l} \phi_{m} \phi_{n} d x, \quad|k| \geq 1 .
$$

An easy computation shows that $G_{i j d l m n}=0$ unless $i \pm j \pm d \pm l \pm m \pm n=0$ for at least one combination of plus and minus signs. Denote $G_{i j d}=G_{i j d i j d}$ and $G_{i}=G_{i i i i i i}$. From [9], if the index set $\mathcal{I}:=\left\{n_{1}<n_{2}<\cdots<n_{b}\right\}$ satisfies

$$
n_{i} \neq n_{j}+n_{d}
$$

for any $i, j, d \in \mathcal{I}$, then

$$
G_{n_{1}}=\cdots=G_{n_{b}}=\frac{5 g_{0}}{2 \pi^{2}}, \quad G_{n_{i} n_{j} n_{j}}=\frac{3 g_{0}}{2 \pi^{2}}, \quad G_{n_{i} n_{j} n_{d}}=\frac{g_{0}}{\pi^{2}}
$$

and

$$
G_{n_{i} n_{j} l}=\frac{g_{0}}{4 \pi^{2}}\left(4-\delta_{n_{i}+l}^{n_{j}}-\delta_{n_{j}+l}^{n_{i}}-\delta_{n_{i}+n_{j}}^{l}\right), \quad G_{n_{i} n_{i} l}=\frac{g_{0}}{4 \pi^{2}}\left(6-\delta_{l}^{2 n_{i}}\right)
$$


hold, where $i \neq j, j \neq d, d \neq i, i, j, d \in\{1,2, \ldots, b\}, l \notin \mathcal{I}$, and for $v \in \mathbb{Z}$,

$$
\delta_{i}^{v}= \begin{cases}1, & i=v \\ 0, & \text { otherwise }\end{cases}
$$

For the convenience, we introduce vectors $\vec{\zeta}=(i, j, d, l, m, n)$. Suppose that

$$
g_{\vec{\zeta}}:=g_{i j d l m n}=\frac{1}{6} G_{i j d l m n}, \quad g_{k, \vec{\zeta}}:=g_{k, i j d l m n}=\frac{1}{6} G_{k, i j d l m n} .
$$

Then $G$ can be expressed as

$$
G=\sum_{i \pm j \pm d \pm l \pm m \pm n=0} g_{\vec{\zeta}} q_{\vec{\zeta}}+\sum_{|k| \geq 1, \vec{\zeta}} g_{k, \vec{\zeta}} e^{\mathrm{i}<k, \vartheta\rangle} q_{\vec{\zeta}}
$$

where $q_{\vec{\zeta}}=q_{i} q_{j} q_{d} \bar{q}_{l} \bar{q}_{m} \bar{q}_{n}$.

We apply the method of [9] to define admissible index sets. For each index set $\mathcal{I}$, define $\Delta_{k}, k=0,1,2,3$, where $\Delta_{k}(k=0,1,2)$ is the set of indices $(i, j, d, l, m, n)$ such that there are exactly $6-k$ components in $\mathcal{I}$, and $\Delta_{3}$ is the set of indices $(i, j, d, l, m, n)$ such that there exist at least three components not in $\mathcal{I}$. We suppose that $\mathcal{I}_{0}=\{(i, j, d, i, j, d)\}$ which means that the last three indices are of the form $(i, j, d)$ or some permutation of it. Define $\mathcal{N}:=\mathcal{I}_{0} \cap \Delta_{0}$ and $\mathcal{M}:=\mathcal{I}_{0} \cap \Delta_{2}$. Then the admissible index set can be defined as follows. It is proved that, in Proposition 2 of [9], there exist infinitely many admissible index sets. For instance, when $b=2$, one can take $\mathcal{I}=\left\{\left(n_{1}, n_{2}\right) \mid n_{1}<n_{2}, n_{1} \equiv 5\right.$ or $9 \bmod (14), n_{2} \equiv 8 \bmod (14), n_{2} \geq$ $\left.11 n_{1}^{2}\right\}$.

Definition 3.1 (Definition 1 in [9]) The index set $\mathcal{I}$ is said to be admissible if and only if $n_{1}, n_{2}, \ldots, n_{b}$ satisfy the following assumptions $\mathrm{A}-\mathrm{C}$ and (14).

A. If $i \pm j \pm d \pm l \pm m \pm n=0, \vec{\zeta} \in \Delta_{0} \backslash \mathcal{N}$, then $\lambda_{i}+\lambda_{j}+\lambda_{d}-\lambda_{l}-\lambda_{m}-\lambda_{n} \neq 0$.

B. If $i \pm j \pm d \pm l \pm m \pm n=0, \vec{\zeta} \in \Delta_{1}$, then $\lambda_{i}+\lambda_{j}+\lambda_{d}-\lambda_{l}-\lambda_{m}-\lambda_{n} \neq 0$.

C. If $i \pm j \pm d \pm l \pm m \pm n=0, \vec{\zeta} \in \Delta_{2} \backslash \mathcal{M}$, then $\lambda_{i}+\lambda_{j}+\lambda_{d}-\lambda_{l}-\lambda_{m}-\lambda_{n} \neq 0$.

Next we transform Hamiltonian (10) into some partial Birkhoff form of order six.

The following lemmas are necessary. We will apply them to proving Proposition 3.1. The proofs of these lemmas are shown in Appendix. Define $\bar{\Delta}:=\Delta_{0} \cup \Delta_{1} \cup \Delta_{2}$ and $\overline{\bar{\Delta}}:=$ $\left(\Delta_{0} \backslash \mathcal{N}\right) \cup \Delta_{1} \cup\left(\Delta_{2} \backslash \mathcal{M}\right)$. Assume that $\eta_{1}>1$ and $0<\eta_{0}<\frac{1}{2}$ are fixed.

Remark 3.1 Here the condition $\eta_{1}>1$ is necessary to obtain the real analyticity of symplectic change. The detailed reason can be found in the proofs of Lemmas 3.1 and 3.2.

Remark 3.2 In order to set the small divisor conditions, we take $\eta_{0}>0$. To ensure the perturbation $\tilde{P}$ small enough, we take $0<\eta_{0}<\frac{1}{2}$.

Lemma 3.1 There is a subset $\underline{\Omega} \subset[\varrho, 2 \varrho]^{\kappa}$ such that every $\omega \in \underline{\Omega}$ satisfies that

$$
|\langle k, \omega\rangle| \geq \frac{\varrho \varepsilon^{\eta_{0}}}{|k|^{\kappa+\eta_{1}}} \quad \text { for all } 0 \neq k \in \mathbb{Z}^{\kappa}
$$


and

$$
\operatorname{meas} \underline{\Omega} \geq \varrho^{\kappa}\left(1-C_{1} \varepsilon^{\eta_{0}}\right)
$$

where the constant $C_{1}$ depends on $\kappa$.

Lemma 3.2 Assume that $(i, j, d, l, m, n) \in \bar{\Delta}$ and $k \neq 0$. Then, when $\varepsilon$ is small enough, there is a subset $\bar{\Omega} \subset[\varrho, 2 \varrho]^{\kappa}$ satisfying that, for any $\omega \in \bar{\Omega}$ and $\lambda_{i}+\lambda_{j}+\lambda_{d}-\lambda_{l}-\lambda_{m}-\lambda_{n} \neq 0$,

$$
\left|\langle k, \omega\rangle+\lambda_{i}+\lambda_{j}+\lambda_{d}-\lambda_{l}-\lambda_{m}-\lambda_{n}\right| \geq \frac{\varrho \varepsilon^{\eta_{0}}}{|k|^{\kappa+\eta_{1}}} .
$$

Moreover,

$$
\text { meas } \bar{\Omega} \geq \varrho^{\kappa}\left(1-C_{2} \varepsilon^{\eta_{0}}\right)
$$

where $C_{2}$ is a constant depending on $\kappa, \varrho, n_{1}$, and $n_{b}$.

Lemma 3.3 For $x_{k} \in \mathbb{C}$ and $k \in \mathbb{Z}^{\kappa}$, if the series $\sum_{k}[k]^{2 \kappa+1}\left|x_{k}\right|^{2}$ converges, then the inequality $\left|\sum_{k} x_{k}\right|^{2} \leq c \sum_{k}[k]^{2 \kappa+1}\left|x_{k}\right|^{2}$ holds, where $[k]=\max \{|k|, 1\},|k|=\left|k_{1}\right|+\left|k_{2}\right|+\cdots+\left|k_{\kappa}\right|$ and $c$ is a constant depending on $\kappa$.

Consider Hamiltonian (10). For each index set $\mathcal{I}$, the following Proposition 3.1 holds.

Proposition 3.1 When $\varepsilon$ is small enough, there exists a subset $\Omega \subset[\varrho, 2 \varrho]^{\kappa}$ with meas $\Omega>$ 0 , and for every $\omega \in \Omega$, there is a real analytic, symplectic change of coordinates $\Psi$ which can transform Hamiltonian (10) into its Birkhoff normal form, i.e.,

$$
H \circ \Psi=\Lambda+\varepsilon \bar{G}+\varepsilon \hat{G}+\varepsilon^{2} K
$$

where

$$
\begin{aligned}
\bar{G}= & \frac{5 g_{0}}{12 \pi^{2}} \sum_{j=1}^{b}\left|q_{n_{j}}\right|^{6}+\frac{9 g_{0}}{4 \pi^{2}} \sum_{i, j=1, \ldots, b, i \neq j}\left|q_{n_{i}}\right|^{4}\left|q_{n_{j}}\right|^{2} \\
& +\frac{6 g_{0}}{\pi^{2}} \sum_{i, j=1, \ldots, b, i \neq j, j \neq d, d \neq i}\left|q_{n_{i}}\right|^{2}\left|q_{n_{j}}\right|^{2}\left|q_{n_{d}}\right|^{2} \\
& +\frac{3}{2} \sum_{l \notin \mathcal{I}} \sum_{i=1, \ldots, b} G_{n_{i} n_{i} l}\left|q_{n_{i}}\right|^{4}\left|q_{l}\right|^{2} \\
& +6 \sum_{l \notin \mathcal{I}} \sum_{i, j=1, \ldots, b, i \neq j} G_{n_{i} n_{j}}\left|q_{n_{i}}\right|^{2}\left|q_{n_{j}}\right|^{2}\left|q_{l}\right|^{2}, \\
\hat{G}= & \sum_{\vec{\zeta} \in \Delta_{3}, i \pm j \pm d \pm l \pm m \pm n=0} g_{\vec{\zeta}} q_{\vec{\zeta}}+\sum_{|k| \geq 1} e^{i \mid\langle k, \vartheta\rangle} \sum_{\vec{\zeta} \in \Delta_{3}} g_{k, \vec{\zeta}} q_{\vec{\zeta}},
\end{aligned}
$$

and $\varepsilon^{\eta_{0}}|K|=\mathcal{O}\left(\left(\|q\|_{a, s}\right)^{10}\right)$. Moreover, the transformation $\Psi$ is defined in a complex neighborhood $D_{1}\left(\frac{\sigma_{1}}{2}\right):=\left\{\vartheta|| \operatorname{Im} \vartheta \mid<\frac{\sigma_{1}}{2}\right\}$ of the tour $\mathbb{T}^{\kappa}$ and a neighborhood of the origin in $l^{a, s}$. 
Proof of Proposition 3.1 Step 1 . We construct the symplectic transformation $\Psi$. Consider a Hamiltonian function

$$
\mathcal{F}=\varepsilon F=\varepsilon \sum_{\vec{\zeta}} F_{\vec{\zeta}} q_{\vec{\zeta}}+\varepsilon \sum_{|k| \geq 1} e^{\mathrm{i}\langle k, \vartheta\rangle} \sum_{\vec{\zeta}} F_{k, \vec{\zeta}} q_{\vec{\zeta}}
$$

with coefficients

$$
\mathrm{i} F_{\vec{\zeta}}= \begin{cases}\frac{g_{\vec{\zeta}}}{\lambda_{i}+\lambda_{j}+\lambda_{d} \lambda_{l}-\lambda_{m}-\lambda_{n}} & \text { if } i \pm j \pm d \pm l \pm m \pm n=0 \text { and } \vec{\zeta} \in \overline{\bar{\Delta}} \\ 0 & \text { otherwise, }\end{cases}
$$

and for $k \neq 0$,

$$
\mathrm{i} F_{k, \vec{\zeta}}= \begin{cases}\frac{g_{k, \vec{\zeta}}}{\langle k, \omega\rangle} & \text { if }|k| \geq 1, \vec{\zeta} \in \bar{\Delta}, \text { and } \\ & \lambda_{i}+\lambda_{j}+\lambda_{d}-\lambda_{l}-\lambda_{m}-\lambda_{n}=0, \\ \frac{g_{k, \vec{\zeta}}}{\langle k, \omega\rangle+\lambda_{i}+\lambda_{j}+\lambda_{d}-\lambda_{l}-\lambda_{m}-\lambda_{n}} & \text { if }|k| \geq 1, \vec{\zeta} \in \bar{\Delta}, \text { and } \\ & \lambda_{i}+\lambda_{j}+\lambda_{d}-\lambda_{l}-\lambda_{m}-\lambda_{n} \neq 0, \\ 0 & \text { otherwise. }\end{cases}
$$

Let $\Psi=X_{\mathcal{F}}^{1}$ be the time-1 map of the vector field of the Hamiltonian $\mathcal{F}$. Expanding at $t=0$ and using Taylor's formula, we obtain

$$
\begin{aligned}
H \circ \Psi= & H+\{H, \mathcal{F}\}+\int_{0}^{1}(1-t)\{\{H, \mathcal{F}\}, \mathcal{F}\} \circ X_{\mathcal{F}}^{t} d t \\
= & \Lambda+\varepsilon G+\varepsilon\{\Lambda, F\} \\
& +\varepsilon^{2}\{G, F\}+\varepsilon^{2} \int_{0}^{1}(1-t)\{\{H, F\}, F\} \circ X_{\mathcal{F}}^{t} d t .
\end{aligned}
$$

For convenience, suppose that

$$
I_{\vec{\zeta}}:=\left[g_{\vec{\zeta}}-\mathrm{i}\left(\lambda_{i}+\lambda_{j}+\lambda_{d}-\lambda_{l}-\lambda_{m}-\lambda_{n}\right) F_{\vec{\zeta}}\right] q_{\vec{\zeta}}
$$

and

$$
I_{k, \vec{\zeta}}:=\left[g_{k, \vec{\zeta}}-\mathrm{i}\left(\langle k, \omega\rangle+\lambda_{i}+\lambda_{j}+\lambda_{d}-\lambda_{l}-\lambda_{m}-\lambda_{n}\right) F_{k, \vec{\zeta}}\right] q_{\vec{\zeta}} .
$$

In the second line of (20), we compute and obtain that

$$
G+\{\Lambda, F\}=\bar{G}+\hat{G}
$$

where

$$
\begin{aligned}
\bar{G}: & =\sum_{\vec{\zeta} \in \mathcal{N} \text { or } \vec{\zeta} \in \mathcal{M} \text { and } i \pm j \pm d \pm l \pm m \pm n=0} I_{\vec{\zeta}} \sum_{\vec{\zeta} \in \mathcal{N} \text { or } \vec{\zeta} \in \mathcal{M} \text { and } i \pm j \pm d \pm l \pm m \pm n=0} g_{\vec{\zeta}} q_{\vec{\zeta}}
\end{aligned}
$$


and

$$
\hat{G}:=\sum_{\vec{\zeta} \in \Delta_{3}} I_{\vec{\zeta}}+\sum_{|k| \geq 1} e^{\mathrm{i}\langle k, \vartheta\rangle} \sum_{\vec{\zeta} \in \Delta_{3}} I_{k, \vec{\zeta}} .
$$

So, (18) and (19) hold true as well as

$$
H \circ \Psi=\Lambda+\varepsilon \bar{G}+\varepsilon \hat{G}+\varepsilon^{2}\{G, F\}+\varepsilon^{2} \int_{0}^{1}(1-t)\{\{H, F\}, F\} \circ X_{\mathcal{F}}^{t} .
$$

Step 2. We prove that $\Psi$ is real analytic.

CLAIM. The vector-field of the Hamiltonian $X_{F}$ is real analytic in a complex neighborhood $\vartheta \in D_{1}\left(\frac{\sigma_{1}}{2}\right)$ of $\mathbb{T}^{\kappa}$ and some neighborhood of the origin in $l^{a, s}$. Furthermore, it satisfies

$$
\left\|F_{\bar{q}}\right\|_{a, s} \leq \frac{C}{\varepsilon^{\eta_{0}}}\left(\|q\|_{a, s}\right)^{5}
$$

The vector-field of $F$ is

$$
X_{F}=\left(0,-\frac{\partial F}{\partial \vartheta}, 2 \mathrm{i} \frac{\partial F}{\partial \bar{q}},-2 \mathrm{i} \frac{\partial F}{\partial q}\right)
$$

Firstly, we discuss $-\frac{\partial F}{\partial \vartheta}$. For $\vartheta \in D_{1}\left(\frac{\sigma_{1}}{2}\right)$ and $q \in l^{a, s}$,

$$
\begin{aligned}
\left|\frac{\partial F}{\partial \vartheta}\right|_{D_{1}\left(\frac{\sigma_{1}}{2}\right)} & =\left|\sum_{|k| \geq 1} e^{\mathrm{i}\langle k, \vartheta\rangle} \mathrm{i} k \sum_{\vec{\zeta} \in \bar{\Delta}} F_{k, \vec{\zeta}} q_{\vec{\zeta}}\right|_{D_{1}\left(\frac{\sigma_{1}}{2}\right)} \\
& \leq \sum_{|k| \geq 1} e^{\frac{|k| \sigma_{1}}{2}}|k| \sum_{\vec{\zeta} \in \bar{\Delta}}\left|F_{k, \vec{\zeta}}\right|\left|q_{\vec{\zeta}}\right| .
\end{aligned}
$$

However, with the even extension of $g$ on $x \in[-\pi, \pi]$, we obtain the Fourier cosine expansion

$$
g_{k}(x)=\sum_{\tau \in \mathbb{Z}} g_{k}^{\tau} \cos \tau x
$$

which together with (15), (13), and Lemma 3.2 yields the estimate of $F_{k, \vec{\zeta}}$ :

$$
\left|F_{k, \vec{\zeta}}\right| \leq C \frac{|k|^{\kappa+\eta_{1}}}{\varepsilon^{\eta_{0}}} \sum_{\tau, \tau+i \pm j \pm d \pm l \pm m \pm n=0}\left|g_{k}^{\tau}\right|
$$

where $C$ is dependent on $\varrho$ and $\mathcal{I}$.

It follows from (23) and (24) that

$$
\begin{aligned}
\left|\frac{\partial F}{\partial \vartheta}\right|_{D_{1}\left(\frac{\sigma_{1}}{2}\right)} \leq & \frac{C}{\varepsilon^{\eta_{0}}} \sum_{|k| \geq 1} e^{\frac{|k| \sigma_{1}}{2}}|k|^{\kappa+\eta_{1}+1} \\
& \times\left(\sum_{\tau+i \pm j \pm d \pm l \pm m \pm n=0, \vec{\zeta} \in \bar{\Delta}}\left|g_{k}^{\tau}\right|\left|q_{i}\right|\left|q_{j}\right|\left|q_{d}\right|\left|\bar{q}_{l}\right|\left|\bar{q}_{m}\right|\left|\bar{q}_{n}\right|\right) .
\end{aligned}
$$


However, from Lemma A.1 in [22], we have

$$
\left|g_{k}^{\tau}\right|<|g|_{\sigma_{1}, 2 a} e^{-|k| \sigma_{1}} e^{-2 a|\tau|}, \quad k \neq 0 .
$$

So

$$
\begin{aligned}
\left|\frac{\partial F}{\partial \vartheta}\right|_{D_{1}\left(\frac{\sigma_{1}}{2}\right)} \leq & \frac{C}{\varepsilon^{\eta_{0}}} \sum_{|k| \geq 1} e^{-\frac{|k| \sigma_{1}}{2}}|k|^{\kappa+\eta_{1}+1} \\
& \times\left(\sum_{\tau+i \pm j \pm d \pm l \pm m \pm n=0, \vec{\zeta} \in \bar{\Delta}} e^{-2 a|\tau|}\left|q_{i}\right|\left|q_{j}\right|\left|q_{d}\right|\left|\bar{q}_{l}\right|\left|\bar{q}_{m}\right|\left|\bar{q}_{n}\right|\right)
\end{aligned}
$$

Suppose that

$$
\tilde{w}=\left(\ldots, \tilde{w}_{-2}, \tilde{w}_{-1}, \tilde{w}_{0}, \tilde{w}_{1}, \tilde{w}_{2}, \ldots\right)
$$

and the component $\tilde{w}_{\tau}=e^{-2 a|\tau|}$ for $\tau \in \mathbb{Z}$, then

$$
\|\tilde{w}\|_{a, s}^{2}=\sum_{\tau}\left|\tilde{w}_{\tau}\right|^{2}[\tau]^{2 s} e^{2 a|\tau|}=\sum_{\tau} e^{-4 a|\tau|}[\tau]^{2 s} e^{2 a|\tau|}=\sum_{\tau} e^{-2 a \tau}[\tau]^{2 s} .
$$

Since $\sum_{\tau} e^{-2 a|\tau|}[\tau]^{2 s}$ is convergent, $\tilde{w} \in l_{b}^{a, s}$. Assuming

$$
w_{j}=\left|q_{j}\right|, \quad w_{-j}=\left|\bar{q}_{j}\right|, \quad w_{0}=0
$$

for $j \geq 1$, it follows from (26) that

$$
\left|\frac{\partial F}{\partial \vartheta}\right|_{D_{1}\left(\frac{\sigma_{1}}{2}\right)} \leq \frac{C}{\varepsilon^{\eta_{0}}} \sum_{|k| \geq 1} e^{-\frac{|k| \sigma_{1}}{2}}|k|^{\kappa+\eta_{1}+1}(\tilde{w} * w * w * w * w * w * w)_{0} .
$$

Since

$$
\sharp\left\{k \in \mathbb{Z}^{\kappa}:|k|=l\right\} \leq 2^{\kappa} l^{\kappa-1}, \quad l \in \mathbb{Z}^{+},
$$

we obtain, from (29) and Lemma 2.2,

$$
\begin{aligned}
\left|\frac{\partial F}{\partial \vartheta}\right|_{D_{1}\left(\frac{\sigma_{1}}{2}\right)} & \leq\|\tilde{w} * w * w * w * w * w * w\|_{a, s} \frac{C}{\varepsilon^{\eta_{0}}} \sum_{l \geq 1} l^{2 \kappa+\eta_{1}} e^{-\frac{l \sigma_{1}}{2}} \\
& \leq \frac{C}{\varepsilon^{\eta_{0}}}\|\tilde{w} * w * w * w * w * w * w\|_{a, s} \sum_{l \geq 1} l^{2 \kappa+\eta_{1}} e^{-\frac{l \tilde{\sigma}_{1}}{2}} \\
& \leq \frac{C}{\varepsilon^{\eta_{0}}}\|q\|_{a, s}^{6} .
\end{aligned}
$$

Secondly, we discuss $\left\|\frac{\partial F}{\partial \bar{q}}\right\|_{a, s} \cdot\left\|\frac{\partial F}{\partial q}\right\|_{a, s}$ can be discussed in the same way.

Since

$$
\left(\left\|F_{\bar{q}}\right\|_{a, s}\right)^{2}=\sum_{l \geq 1}\left|F_{\bar{q}_{l}}\right|^{2} e^{2 a l} l^{2 s},
$$


we need to estimate $\left|F_{\bar{q}_{l}}\right|^{2}$. It is evident that

$$
\left|F_{\bar{q}_{l}}\right|^{2} \leq C\left(F_{0 l}\right)^{2}+C\left(F_{1 l}\right)^{2}
$$

where

$$
F_{0 l}=\left|\sum_{i \pm j \pm d \pm l \pm m \pm n=0, \vec{\zeta} \in \overline{\bar{\Delta}}} F_{\vec{\zeta}} q_{i} q_{j} q_{d} \bar{q}_{m} \bar{q}_{n}\right|
$$

and

$$
F_{1 l}=\left|\sum_{|k| \geq 1} e^{\mathrm{i}\langle k, \vartheta\rangle} \sum_{\vec{\zeta} \in \bar{\Delta}} F_{k, \vec{\zeta}} q_{i} q_{j} q_{d} \bar{q}_{m} \bar{q}_{n}\right| .
$$

For $F_{\vec{\zeta}}$, according to the definition of admissible set, the divisor

$$
\delta:=\lambda_{i}+\lambda_{j}+\lambda_{d}-\lambda_{l}-\lambda_{m}-\lambda_{n} \neq 0 .
$$

So, $|\delta| \geq 1$ holds for all $\vec{\zeta} \in \overline{\bar{\Delta}}$. Therefore,

$$
F_{0 l}^{2} \leq C\left(\sum_{\vec{\zeta} \in \overline{\bar{\Delta}}, \pm i \pm j \pm d \pm m \pm n=l}\left|q_{i} q_{j} q_{d} \bar{q}_{m} \bar{q}_{n}\right|\right)^{2} .
$$

It follows from (28) that

$$
F_{0 l}^{2} \leq C\left((w * w * w * w * w)_{l}\right)^{2} .
$$

So, using Lemma 2.2, we have

$$
\begin{aligned}
\sum_{l \geq 1} F_{0 l}^{2} l^{2 s} e^{2 a l} & \leq C \sum_{|l| \geq 1}\left|(w * w * w * w * w)_{l}\right|^{2}|l|^{2 s} e^{2 a|l|} \\
& \leq C\left(\|w * w * w * w * w\|_{a, s}\right)^{2} \leq C\left(\|q\|_{a, s}^{5}\right)^{2},
\end{aligned}
$$

where $C$ depends on $g$ and $\mathcal{I}$.

Supposing that $\Omega=\bar{\Omega} \cap \underline{\Omega}$, according to Lemmas 3.1 and 3.2,

$$
\text { meas } \Omega \geq \varrho^{\kappa}\left(1-C \varepsilon^{\eta_{0}}\right)
$$

holds and meas $\Omega>0$ when $\varepsilon$ is small enough. In the following, we assume $\omega \in \Omega$. Thus (16) and (17) are true. Using Lemma 3.3 and (24),

$$
\begin{aligned}
F_{1 l}^{2} & \leq C \sum_{|k| \geq 1}[k]^{2 \kappa+1}\left|e^{\mathrm{i}\langle k, \vartheta\rangle}\right|^{2}\left(\sum_{\vec{\zeta} \in \bar{\Delta}}\left|F_{k, \bar{\zeta}}\right|\left|q_{i} q_{j} q_{d} \bar{q}_{m} \bar{q}_{n}\right|\right)^{2} \\
& \leq \frac{C}{\varepsilon^{2 \eta_{0}}} \sum_{|k| \geq 1}[k]^{4 \kappa+2 \eta_{1}+1}\left|e^{\mathrm{i}\langle k, \vartheta\rangle}\right|^{2}
\end{aligned}
$$




$$
\times\left(\sum_{\tau+i \pm j \pm d \pm l \pm m \pm n=0, \bar{\zeta} \in \bar{\Delta}}\left|g_{k}^{\tau}\right|\left|q_{i}\right|\left|q_{j}\right|\left|q_{d}\right|\left|\bar{q}_{m}\right|\left|\bar{q}_{n}\right|\right)^{2} .
$$

From (25), we have

$$
\begin{aligned}
F_{1 l}^{2} \leq & \frac{C}{\varepsilon^{2 \eta_{0}}} \sum_{|k| \geq 1}[k]^{4 \kappa+2 \eta_{1}+1}\left|e^{\mathrm{i}\langle k, \vartheta\rangle}\right|^{2} e^{-2|k| \sigma_{1}} \\
& \times\left(\sum_{\tau+i \pm j \pm d \pm l \pm m \pm n=0, \vec{\zeta} \in \bar{\Delta}} e^{-2 a|\tau|}\left|q_{i}\right|\left|q_{j}\right|\left|q_{d}\right|\left|\bar{q}_{m}\right|\left|\bar{q}_{n}\right|\right)^{2} .
\end{aligned}
$$

It is derived from (27) and (28) that

$$
F_{1 l}^{2} \leq \frac{C}{\varepsilon^{2 \eta_{0}}} \sum_{|k| \geq 1}[k]^{4 \kappa+2 \eta_{1}+1}\left|e^{\mathrm{i} \mid(k, \vartheta)}\right|^{2} e^{-2|k| \sigma_{1}}\left((\tilde{w} * w * w * w * w * w)_{l}\right)^{2} .
$$

For all $(\vartheta, x) \in D_{1}\left(\sigma_{1} / 2\right) \times D_{2}(2 a)$,

$$
\begin{aligned}
F_{1 l}^{2} & \leq \frac{C}{\varepsilon^{2 \eta_{0}}} \sum_{|k| \geq 1}[k]^{4 \kappa+2 \eta_{1}+1} e^{\sigma_{1}|k|} e^{-2|k| \sigma_{1}}\left((\tilde{w} * w * w * w * w * w)_{l}\right)^{2} \\
& \leq \frac{C}{\varepsilon^{2 \eta_{0}}}\left((\tilde{w} * w * w * w * w * w)_{l}\right)^{2} \sum_{|k| \geq 1}[k]^{4 \kappa+2 \eta_{1}+1} e^{-|k| \sigma_{1}}
\end{aligned}
$$

Since (30) holds, we obtain, from (34),

$$
\begin{aligned}
F_{1 l}^{2} & \leq \frac{C}{\varepsilon^{2 \eta_{0}}}\left((\tilde{w} * w * w * w * w * w)_{l}\right)^{2} \sum_{j \geq 1} j^{5 \kappa+2 \eta_{1}} e^{-j \sigma_{1}} \\
& \leq \frac{C}{\varepsilon^{2 \eta_{0}}}\left((\tilde{w} * w * w * w * w * w)_{l}\right)^{2} \sum_{j \geq 1} j^{5 \kappa+2 \eta_{1}} e^{-j \tilde{\sigma}_{1}} .
\end{aligned}
$$

Therefore,

$$
\begin{aligned}
\sum_{l \geq 1} F_{1 l}^{2} l^{2 s} e^{2 a l} & \leq \frac{C}{\varepsilon^{2 \eta_{0}}} \sum_{|l| \geq 1}|(\tilde{w} * w * w * w * w * w)|^{2}|l|^{2 s} e^{2 a|l|} \\
& \leq \frac{C}{\varepsilon^{2 \eta_{0}}}\left(\|\tilde{w} * w * w * w * w * w\|_{a, s}\right)^{2} \leq \frac{C}{\varepsilon^{2 \eta_{0}}}\left(\|q\|_{a, s}^{5}\right)^{2},
\end{aligned}
$$

which together with (31), (32), and (33) yields that

$$
\left(\left\|F_{\bar{q}}\right\|_{a, s}\right)^{2} \leq C \sum_{l \geq 1}\left(F_{0 l}^{2}+F_{1 l}^{2}\right) l^{2 s} e^{2 a l} \leq \frac{C}{\varepsilon^{2 \eta_{0}}}\left(\|q\|_{a, s}^{5}\right)^{2}
$$

for $0<\varepsilon<1$, where the constant $C$ depends on $\varrho, g, \tilde{\sigma}_{1}, a, \kappa, \mathcal{I}, \eta_{1}$, and $s$. The analyticity of $X_{F}$ follows from the analyticity of each component function and its local boundedness. The claim is proved.

Step 3. Similar to the proof of (22), we attain that $|\hat{G}|=\mathcal{O}\left(\|q\|_{a, s}^{6}\right)$. Finally, we estimate $K$. Suppose that

$$
K=\{G, F\}+\int_{0}^{1}(1-t)\{\{H, F\}, F\} \circ X_{\mathcal{F}}^{t} d t .
$$


By using Lemma 2.3 and (22), and from the fact that

$$
|\{\Lambda, F\}|=|\bar{G}+\hat{G}-G|=\mathcal{O}\left(\|q\|_{a, s}^{6}\right)
$$

we obtain

$$
|\{G, F\}| \leq \frac{C}{\varepsilon^{\eta_{0}}}\left(\|q\|_{a, s}\right)^{10} \text { and } \quad|\{\{\Lambda, F\}, F\}| \leq \frac{C}{\varepsilon^{\eta_{0}}}\left(\|q\|_{a, s}\right)^{10} \text {. }
$$

Furthermore,

$$
|\{\{G, F\}, F\}| \leq \frac{C}{\varepsilon^{2 \eta_{0}}}\left(\|q\|_{a, s}\right)^{14}
$$

and (7) hold. Hence, $|K| \leq \frac{C}{\varepsilon^{\eta 0}}\left(\|q\|_{a, s}\right)^{10}$ for $\|q\|_{a, s} \leq 1$. This completes the proof.

We introduce the symplectic polar and complex coordinates by setting

$$
\begin{cases}q_{n_{j}}=\sqrt{2 \varsigma_{j}+2 I_{j}} e^{-\mathrm{i} \theta_{j}}, & 1 \leq j \leq b \\ q_{j}=\sqrt{2} w_{j}, & j \in \mathbb{Z}_{1}\end{cases}
$$

where $\varsigma_{j} \in[0,1]$ and $\mathbb{Z}_{1}:=\mathbb{Z}^{+} \backslash \mathcal{I}$. Then the Hamiltonian is changed to

$$
H=\sum_{1 \leq i \leq \kappa} \omega_{i} J_{i}+\sum_{1 \leq j \leq b} \varpi_{j} I_{j}+\sum_{l \in \mathbb{Z}_{1}} \hat{\Omega}_{l} w_{l} \bar{w}_{l}+P
$$

with symplectic structure $\sum_{1 \leq i \leq \kappa} d \vartheta_{i} \wedge d J_{i}+\sum_{1 \leq j \leq b} d \theta_{j} \wedge d I_{j}+i \sum_{l \in \mathbb{Z}_{1}} d w_{l} \wedge d \bar{w}_{l}$, where $P=\varepsilon \breve{G}+\varepsilon \hat{G}+\varepsilon^{2} K$,

$$
\begin{aligned}
\breve{G}= & \frac{10 g_{0}}{3 \pi^{2}} \sum_{j=1}^{b}\left(I_{j}^{3}+3 I_{j}^{2} \varsigma_{j}+\varsigma_{j}^{3}\right)+\frac{18 g_{0}}{\pi^{2}} \sum_{i \neq j, i, j=1, \ldots, b}\left(\varsigma_{j} \varsigma_{i}^{2}+\varsigma_{j} I_{i}^{2}+2 \varsigma_{i} I_{i} I_{j}+I_{j} I_{i}^{2}\right) \\
& +\frac{48 g_{0}}{\pi^{2}} \sum_{i \neq j, j \neq d, d \neq i, i, j, d=1, \ldots, b}\left(\varsigma_{i} \varsigma_{j} \varsigma_{d}+\varsigma_{i} I_{j} I_{d}+I_{i} \varsigma_{j} I_{d}+I_{i} I_{j} \varsigma_{d}+I_{i} I_{j} I_{d}\right) \\
& +12 \sum_{l \in \mathbb{Z}_{1}} \sum_{i=1}^{b} G_{n_{i} n_{i} l} I_{i}^{2}\left|w_{l}\right|^{2}+48 \sum_{l \in \mathbb{Z}_{1}} \sum_{i \neq j, i, j=1, \ldots, b} G_{n_{i} n_{j} l} I_{i} I_{j}\left|w_{l}\right|^{2}, \\
\varpi_{j}= & \lambda_{j}+\frac{2 g_{0} \varepsilon}{\pi^{2}}\left(5 \varsigma_{j}^{2}+18 \sum_{i \neq j, i=1, \ldots, b} \varsigma_{i} \varsigma_{j}+9 \sum_{i \neq j, i=1, \ldots, b} \varsigma_{i}^{2}\right. \\
& \left.+72 \sum_{i \neq j, i \neq d, d \neq j, i, d=1, \ldots, b} \varsigma_{i} \varsigma_{d}\right), \quad j=1, \ldots, b,
\end{aligned}
$$

and

$$
\hat{\Omega}_{l}=\lambda_{l}+12 \varepsilon \sum_{i=1}^{b} G_{n_{i} n_{i} l} \varsigma_{i}^{2}+48 \varepsilon \sum_{i \neq j, i, j=1, \ldots, b} G_{n_{i} n_{j} l} \varsigma_{i} \varsigma_{j}, \quad l \in \mathbb{Z}_{1} .
$$


For any $\omega_{-} \in \Omega$ fixed and $\omega \in \overline{\bar{\Omega}}:=\left\{\omega \in \Omega|| \omega-\omega_{-} \mid \leq \varepsilon^{\frac{7}{6}}\right\}$, we introduce the new parameter $\bar{\omega}$ as follows:

$$
\omega=\omega_{-}+\varepsilon^{\frac{7}{6}} \bar{\omega}, \quad \bar{\omega} \in[0,1]^{\kappa} .
$$

Clearly, $\overline{\bar{\Omega}} \times[0,1]^{b} \subset \Omega \times[0,1]^{b}$.

Scaling the variables as follows:

$$
\left\{\begin{array}{l}
\bar{\omega}=\varepsilon^{\frac{1}{6}} \tilde{\bar{\omega}}, \\
\varsigma=\varepsilon^{\frac{1}{6}} \tilde{\zeta} \\
w=\varepsilon^{\frac{1}{12}} \tilde{w} \\
\bar{w}=\varepsilon^{\frac{1}{12}} \overline{\tilde{w}} \\
I=\varepsilon^{\frac{1}{6}} \tilde{I}, \\
J=\varepsilon^{\frac{1}{\zeta}} \tilde{J},
\end{array}\right.
$$

one can obtain a Hamiltonian system given by the rescaled Hamiltonian

$$
\begin{aligned}
\tilde{H} & =\varepsilon^{-\frac{1}{2}} H\left(\vartheta, \varepsilon^{\frac{1}{6}} \tilde{J}, \theta, \varepsilon^{\frac{1}{6}} \tilde{I}, \varepsilon^{\frac{1}{12}} \tilde{w}, \varepsilon^{\frac{1}{12}} \overline{\tilde{w}}, \varepsilon^{\frac{1}{6}} \tilde{\bar{\omega}}, \varepsilon^{\frac{1}{6}} \tilde{\zeta}\right) \\
& =\sum_{1 \leq i \leq m} \tilde{\omega}_{i} \tilde{J}_{i}+\sum_{1 \leq j \leq b} \tilde{\tilde{\omega}}_{j} \tilde{I}_{j}+\sum_{l \in \mathbb{Z}_{1}} \tilde{\hat{\Omega}}_{l} \tilde{w}_{l} \overline{\mathcal{w}_{l}}+\tilde{P}
\end{aligned}
$$

where

$$
\begin{aligned}
\tilde{\omega}_{i}(\tilde{\bar{\omega}})= & \varepsilon^{-\frac{1}{3}} \omega_{-i}+\varepsilon \tilde{\bar{\omega}}_{i}, \quad 1 \leq i \leq \kappa, \\
\tilde{\omega}_{j}(\tilde{\zeta})= & \varepsilon^{-\frac{1}{3}} \lambda_{n_{j}}+\frac{2 g_{0} \varepsilon}{\pi^{2}}\left(5 \tilde{\zeta}_{j}^{2}+18 \sum_{i \neq j, i=1, \ldots, b} \tilde{\zeta}_{i} \tilde{\zeta}_{j}+9 \sum_{i \neq j, i=1, \ldots, b} \tilde{\zeta}_{i}^{2}\right. \\
& \left.+72 \sum_{i \neq d, d \neq j, i \neq j i, d=1, \ldots, b} \tilde{\zeta}_{i} \tilde{\zeta}_{d}\right), \quad j=1, \ldots, b, \\
\tilde{\hat{\Omega}}_{l}(\tilde{\zeta})= & \varepsilon^{-\frac{1}{3}} \lambda_{l}+12 \varepsilon \sum_{i=1}^{b} G_{n_{i} n_{i}} \tilde{\zeta}_{i}^{2}+48 \varepsilon \sum_{i \neq j, i, j=1, \ldots, b} G_{n_{i} n_{j} l} \tilde{\zeta}_{i} \tilde{\zeta}_{j}, \quad l \in \mathbb{Z}_{1} .
\end{aligned}
$$

Clearly,

$$
\omega=\varepsilon^{\frac{1}{3}} \tilde{\omega}, \quad \varpi=\varepsilon^{\frac{1}{3}} \tilde{\varpi}, \quad \hat{\Omega}=\varepsilon^{\frac{1}{3}} \tilde{\hat{\Omega}} .
$$

Next, we introduce some notations. Assume that

$$
D(\sigma, r):=\left\{(\vartheta, \tilde{J}, \theta, \tilde{I}, \tilde{w}, \tilde{\bar{w}}):|\operatorname{Im} \vartheta|<\sigma,|\operatorname{Im} \theta|<\sigma,|\tilde{I}|<r^{2},\|\tilde{w}\|_{a, s}<r,\|\overline{\tilde{w}}\|_{a, s}<r\right\}
$$

is a complex neighborhood of $\mathbb{T}^{\kappa} \times\{\tilde{J}=0\} \times \mathbb{T}^{b} \times\{\tilde{I}=0\} \times\{\tilde{w}=0\} \times\{\overline{\tilde{w}}=0\}$, where $|\cdot|$ denotes the sup-norm for complex vectors. For $p(p \geq 1)$ order Whitney smooth function $F(\xi)$, define

$$
\|F\|_{\Pi}^{*}=\max \left\{\sup _{\xi \in \Pi}|F|, \ldots, \sup _{\xi \in \Pi}\left|\frac{\partial^{p} F}{\partial \xi^{p}}\right|\right\}
$$


and

$$
\|F\|_{* \Pi}=\max \left\{\sup _{\xi \in \Pi}\left|\frac{\partial F}{\partial \xi}\right|, \ldots, \sup _{\xi \in \Pi}\left|\frac{\partial^{p} F}{\partial \xi^{p}}\right|\right\} .
$$

If $F(\xi)$ is a vector function from $\xi$ to $l^{a, s}\left(\mathbb{R}^{n}\right)$, which is $p$ order Whitney smooth on $\xi$, define $\|F\|_{a, s, \Pi}^{*}=\left\|\left(\left\|F_{i}(\xi)\right\|_{\Pi}^{*}\right)_{i}\right\|_{a, s}\left(\|F\|_{\mathbb{R}^{n}, \Pi}^{*}=\max _{i}\left(\left\|F_{i}(\xi)\right\|_{\Pi}^{*}\right)\right)$. If $F(\eta, \xi)$ is a vector function from $D(\sigma, r) \times \Pi$ to $l^{a, s}$, define $\|F\|_{a, s, D \times \Pi}^{*}=\sup _{\eta \in D(\sigma, r)}\|F\|_{a, s, \Pi}^{*}$. We usually omit $D$ for brevity. For functions $F$, a corresponding Hamiltonian vector field is defined as $X_{F}=\left(F_{\tilde{\tilde{J}}},-F_{\vartheta}, F_{\tilde{I}},-F_{\theta}, i F_{\tilde{\tilde{w}}},-i F_{\tilde{w}}\right)^{T}$. Denote the weighted norm for $X_{F}$ by letting

$$
\left|X_{F}\right|_{r, D(\sigma, r) \times \Pi}^{*}=\frac{1}{r^{2}}\left\|F_{\vartheta}\right\|_{\Pi}^{*}+\frac{1}{r^{2}}\left\|F_{\theta}\right\|_{\Pi}^{*}+\left\|F_{\tilde{I}}\right\|_{\Pi}^{*}+\frac{1}{r}\left\|F_{\tilde{w}}\right\|_{a, s, \Pi}^{*}+\frac{1}{r}\left\|F_{\tilde{\tilde{w}}}\right\|_{a, s, \Pi}^{*} .
$$

In the following, we suppose $p=2$. Fix $\sigma_{0}=\frac{\sigma_{1}}{2}$ and $r=r_{0}$, where $0<r_{0}<1$ and $r_{0}$ is fixed. On $D\left(\sigma_{0}, r_{0}\right)$ and for $\tilde{\xi}=(\tilde{\bar{\omega}}, \tilde{\zeta}) \in\left[\frac{\beta}{2}, 3 \beta\right]^{\kappa+b}$, from Prop. 3.1 we can get that

$$
|\tilde{P}| \leq|\varepsilon \tilde{\hat{G}}|+|\varepsilon \tilde{\tilde{G}}|+\left|\varepsilon^{2} \tilde{K}\right| \leq C \varepsilon^{\frac{7}{8}}
$$

Using Cauchy estimates, we have that

$$
\left|X_{\tilde{P}}\right|_{r, D(\sigma, r) \times \Pi}^{*} \leq \frac{C}{\sigma} \varepsilon^{\frac{7}{8}} \beta^{-2}
$$

on $D(\sigma, r)$ with $\sigma=\min \left\{\frac{\sigma_{0}}{2}, \frac{1}{2}\right\}, r=\frac{r_{0}}{2}, \Pi=[\beta, 2 \beta]^{\kappa+b}$, and $\beta$ will be denoted later. Since $(\bar{\omega}, \varsigma) \in[0,1]^{k+b}$, we assume

$$
3 \beta<\varepsilon^{-\frac{1}{6}} .
$$

For simplicity, we still denote $\tilde{H}$ by $H, \tilde{J}$ by $J, \tilde{I}$ by $I, \tilde{w}$ by $w, \overline{\tilde{w}}$, by $\bar{w}, \tilde{\omega}$ by $\omega, \tilde{\varpi}$ by $\varpi, \tilde{\hat{\Omega}}$ by $\hat{\Omega}, \tilde{\bar{\omega}}$ by $\bar{\omega}, \tilde{\zeta}$ by $\varsigma$, and $\tilde{P}$ by $P$.

Remark 3.3 Actually, we eventually take $\beta=\varepsilon^{-\frac{1}{12}}$ as $\varepsilon$ is small enough. The reason can be found in Sect. 4.1.

\section{An infinite-dimensional KAM theorem}

Consider small perturbations of an infinite-dimensional Hamiltonian in the parameter dependent normal form

$$
N=\sum_{1 \leq i \leq \kappa} \omega_{i}(\bar{\omega}) J_{i}+\sum_{1 \leq j \leq b} \varpi_{j}(\varsigma) I_{j}+\sum_{l \in \mathbb{Z}_{1}} \hat{\Omega}_{l}(\varsigma) w_{l} \bar{w}_{l}
$$

on a phase space

$$
\mathcal{P}^{a, s}=\mathbb{T}^{\kappa} \times \mathbb{R}^{\kappa} \times \mathbb{T}^{b} \times \mathbb{R}^{b} \times l^{a, s} \times l^{a, s} \ni(\vartheta, J, \theta, I, w, \bar{w}),
$$

where

$$
\omega_{i}=\frac{\omega_{-i}}{\varepsilon^{\frac{1}{3}}}+\mathcal{O}\left(\bar{\omega}^{1}\right), \quad \varpi_{j}=\frac{j^{2}+\cdots}{\varepsilon^{\frac{1}{3}}}+\mathcal{O}\left(\varsigma^{2}\right), \quad \hat{\Omega}_{l}=\frac{l^{2}+\cdots}{\varepsilon^{\frac{1}{3}}}+\mathcal{O}\left(\varsigma^{2}\right),
$$


$a>0, s>1 / 2$; the dots stand for finite lower order terms of $j$ and $l$, respectively; $\omega_{-} \in$ $\Omega$ is a constant vector; and $\mathcal{O}\left(\bar{\omega}^{p}\right)$ and $\mathcal{O}\left(\varsigma^{p}\right)$ mean $p$ th order terms in $\bar{\omega}_{1}, \ldots, \bar{\omega}_{\kappa}$ and $\varsigma_{1}, \ldots, \varsigma_{b}$, respectively. Denote $\Pi_{1}=[\beta, 2 \beta]^{\kappa}, \Pi_{2}=[\beta, 2 \beta]^{b}$, and $\Pi:=\Pi_{1} \times \Pi_{2}$. Suppose that $\|\omega\|_{*, \Pi_{1}} \leq M_{11},\|\varpi\|_{*, \Pi_{2}} \leq M_{12},\left\|\hat{\Omega}_{j}\right\|_{*, \Pi_{2}} \leq M_{2}$, and $\max \left\{M_{11}, M_{12}\right\}+M_{2} \geq 1$. Define $M=\left(\max \left\{M_{11}, M_{12}\right\}+M_{2}\right)^{2}$.

For the Hamiltonian $H=N+P$, there exists $\kappa+b$-dimensional, linearly stable torus $\mathcal{T}_{0}^{\kappa+b}=\mathbb{T}^{\kappa} \times\{0\} \times \mathbb{T}^{b} \times\{0,0,0\}$ with frequencies $\hat{\omega}=(\omega(\bar{\omega}), \varpi(\varsigma))$ when $P=0$, where

$$
\hat{\omega}_{i}= \begin{cases}\omega_{i}(\bar{\omega}), & 1 \leq i \leq \kappa \\ \varpi_{i-\kappa}(\varsigma), & \kappa+1 \leq i \leq \kappa+b\end{cases}
$$

Our aim is to prove the persistence of a large portion of this family of linearly stable rotational tori under small perturbations. Suppose that the perturbation $P$ is real analytic in the space variables, $C^{2}$ in $(\bar{\omega}, \varsigma)$, and for each $\xi=(\bar{\omega}, \varsigma) \in \Pi$ its Hamiltonian vector field $X_{P}=\left(P_{J},-P_{\vartheta}, P_{I},-P_{\theta}, i P_{\bar{w}},-i P_{w}\right)^{T}$ defines near $\mathcal{T}_{0}^{\kappa+b}$ a real analytic map $X_{P}: \mathcal{P}^{a, s} \rightarrow \mathcal{P}^{a, s}$. Under the above assumptions, we have the following theorem.

Theorem 4.1 Suppose that $H=N+P$ satisfies

$$
\epsilon=\left|X_{P}\right|_{r, D(\sigma, r) \times \Pi}^{*} \leq \gamma \sigma^{2(1+\mu)}
$$

where $\gamma$ depends on $\kappa, b, \tau$ and $M, \mu=3 \tau+2+(\kappa+b) / 2$. Then there exists a Cantor set $\tilde{\Pi}_{\epsilon} \subset \Pi, a$ Whitney smooth family of torus embedding $\Phi: \mathbb{T}^{\kappa+b} \times \tilde{\Pi}_{\epsilon} \rightarrow \mathcal{P}^{a, s}$ and a Whitney smooth map $\tilde{\hat{\omega}}_{*}=\left(\tilde{\omega}_{*}, \tilde{\varpi}_{*}\right): \tilde{\Pi}_{\epsilon} \rightarrow \mathbb{R}^{\kappa+b}$ such that, for each $\xi \in \tilde{\Pi}_{\epsilon}$, the map $\Phi$ restricted to $\mathbb{T}^{\kappa+b} \times\{\xi\}$ is a real analytic embedding of a rotational torus with frequencies $\tilde{\hat{\omega}}_{*}(\xi)$ for the Hamiltonian $\mathrm{H}$ at $\xi$.

Each embedding is real analytic on $|\operatorname{Im} \vartheta|<\frac{\sigma}{2}$ and $|\operatorname{Im} \theta|<\frac{\sigma}{2}$, and

$$
\left|\Phi-\Phi_{0}\right|_{r}^{*} \leq c \epsilon^{\frac{1}{2}}, \quad\left\|\tilde{\hat{\omega}}_{*}-\tilde{\hat{\omega}}\right\|^{*} \leq c \epsilon
$$

uniformly on that domain and $\tilde{\Pi}_{\epsilon}$, where $\Phi_{0}$ is the trivial embedding $\mathbb{T}^{\kappa+b} \times \Pi \rightarrow \mathcal{T}_{0}^{\kappa+b}$.

Remark 4.1 In fact, the parameter $\tau>2 \kappa+2 b+5$ is required. The reason can be found in the proofs of Lemmas 4.2 and 4.3 .

Remark 4.2 The regularity of the vector-field is $X_{P}: \mathcal{P}^{a, s} \rightarrow \mathcal{P}^{a, s^{\prime}}$ with $s^{\prime}=s$. In this theorem, one cannot expect $s^{\prime}>s$, since the original equation explicitly contains $x$.

\section{Assume}

$$
\varepsilon \beta<1 .
$$

From (36), (37), and (38), noticing $b \geq 2$, we get that $M_{11}=1, M_{12}=\frac{2\left|g_{0}\right|(144 b-252)}{\pi^{2}}$ and $M_{2}=\frac{\left|g_{0}\right|(96 b-24)}{\pi^{2}}$. Obviously, $\max \left\{M_{11}, M_{12}\right\} \geq 1$ and $M_{2}>0$. So, we can take $M=$ $\left(\max \left\{M_{11}, M_{12}\right\}+M_{2}\right)^{2}$. Note that $M$ is independent of $\varepsilon$. 
Assuming

$$
\beta^{-2}<1
$$

we have that

$$
\left|X_{P}\right|_{r, D(r, \sigma)}^{*}=\epsilon \leq \frac{\varepsilon^{\frac{3}{4}}}{\sigma}
$$

as $\varepsilon$ is small enough. According to the assumption, we need

$$
\frac{\varepsilon^{\frac{3}{4}}}{\sigma} \leq \gamma \sigma^{2(1+\mu)}
$$

So, we can take $\varepsilon \leq\left(\gamma \sigma^{3+2 \mu}\right)^{\frac{4}{3}}$. According to the reference [9], one can take $\gamma=$

$\frac{\gamma_{0}^{6}}{20^{2(1+\mu)\left(c M^{2}\right)^{6}}}$, where $\gamma_{0} \leq \frac{1}{c(M+1)^{2} 2^{12+4 \mu}}$. Therefore, $\varepsilon \leq\left(\frac{\gamma_{0}^{6}}{20^{20(1+\mu)\left(c M^{2}\right)^{6}}} \sigma^{2 \mu+3}\right)^{\frac{4}{3}}$.
Suppose $\mathbf{x}=(\vartheta, \theta)$ and $\mathbf{y}=(J, I)$, where

$$
\mathbf{x}_{i}=\left\{\begin{array}{ll}
\vartheta_{i}, & 1 \leq i \leq \kappa, \\
\theta_{i-\kappa}, & \kappa+1 \leq i \leq \kappa+b
\end{array} \quad \text { and } \quad \mathbf{y}_{i}= \begin{cases}J_{i}, & 1 \leq i \leq \kappa \\
I_{i-\kappa}, & \kappa+1 \leq i \leq \kappa+b\end{cases}\right.
$$

We take $\xi \in \Pi$. Then Hamiltonian can be written as

$$
H=N+P=\sum_{1 \leq i \leq \kappa+b} \hat{\omega}_{i}(\xi) \mathbf{y}_{i}+\sum_{l \in \mathbb{Z}_{1}} \hat{\Omega}_{l}(\xi) w_{l} \bar{w}_{l}+P(\mathbf{x}, \mathbf{y}, w, \bar{w}, \xi)
$$

Using exactly the same KAM procedure with [9], we can prove this theorem. The proof is standard and the detailed steps can be found in [9]. In every KAM iteration step, some parameter sets are thrown (see [9]). So, it is necessary to identify that the measure of $\tilde{\Pi}_{\epsilon}$ is positive. In order to settle this problem, one can estimate the measure of the thrown parameters sets. We compute and attain that the total measure of these parameters sets, namely $\tilde{\Pi}$, is $\mathcal{O}\left(\varepsilon^{-\frac{15}{16}}\right)$. When $\varepsilon$ is small enough, we can have meas $\tilde{\Pi}<\varepsilon^{-1}$. The details can be found in [9]. For clarity, we show the measure estimate in the first KAM iteration step and put the proof in Sect. 4.1.

We take $\beta=\varepsilon^{-\frac{1}{12}}$ in terms of (39), (40), (41), and (43). So meas $\Pi=\varepsilon^{-\frac{\kappa+b}{12}}$. If $\kappa+b>12$, the meas $\tilde{\Pi}_{\epsilon}>0$, where $\tilde{\Pi}_{\epsilon}:=\Pi \backslash \tilde{\Pi}$. Therefore, there exists a Cantor set $\tilde{\Pi}_{\epsilon} \subset \Pi$ with positive measure such that, for each $\xi \in \tilde{\Pi}_{\epsilon}$, the map $\Phi$ restricted to $\mathbb{T}^{\kappa+b} \times\{\xi\}$ is a real analytic embedding of a rotational torus with frequencies $\tilde{\hat{\omega}}_{*}(\xi)$ for the Hamiltonian $H$ at $\xi$. By applying Theorem 4.1, we get Theorem 2.1.

Remark 4.3 By using the method of this paper, one cannot expect quasi-periodic solutions for $\kappa+b \leq 12$. This is mainly because when $\kappa+b$ reduces, meas $\Pi=\varepsilon^{-\frac{\kappa+b}{12}}$ reduces. But meas $\tilde{\Pi}<\varepsilon^{-1}$. So meas $\tilde{\Pi}_{\epsilon}$ may be not positive.

\subsection{Measure estimates in the first step}

The thrown parameter sets in the first step are

$$
\left(\bigcup_{k \neq 0} A_{k 1}^{1}\right) \cup\left(\bigcup_{k}\left(A_{k 2}^{1} \cup A_{k 3}^{1} \cup A_{k 4}^{1}\right)\right)
$$


where

$$
\begin{aligned}
& A_{k 1}^{1}=\left\{\xi \in \Pi:|\langle k, \hat{\omega}\rangle|<\frac{\epsilon^{\frac{1}{6}}}{A_{k}}\right\}, \\
& A_{k 2}^{1}=\bigcup_{i \in \mathbb{Z}_{1}} B_{k i}^{1,1}=\bigcup_{i \in \mathbb{Z}_{1}}\left\{\xi \in \Pi:\left|\langle k, \hat{\omega}\rangle+\hat{\Omega}_{i}\right|<\frac{\epsilon^{\frac{1}{6}}}{A_{k}}\right\}, \\
& A_{k 3}^{1}=\bigcup_{i, j \in \mathbb{Z}_{1}} B_{k i j}^{1,11}=\bigcup_{i, j \in \mathbb{Z}_{1}}\left\{\xi \in \Pi:\left|\langle k, \hat{\omega}\rangle+\hat{\Omega}_{i}+\hat{\Omega}_{j}\right|<\frac{\epsilon^{\frac{1}{6}}(|i-j|+1)}{A_{k}}\right\}, \\
& A_{k 4}^{1}=\bigcup_{i, j \in \mathbb{Z}_{1}, i \neq j} B_{k i j}^{1,12}=\bigcup_{i, j \in \mathbb{Z}_{1}, i \neq j}\left\{\xi \in \Pi:\left|\langle k, \hat{\omega}\rangle+\hat{\Omega}_{i}-\hat{\Omega}_{j}\right|<\frac{\epsilon^{\frac{1}{6}}(|i-j|+1)}{A_{k}}\right\},
\end{aligned}
$$

where $A_{k}=1+|k|^{\tau}$. Clearly, meas $\left(A_{02}^{1} \cup A_{03}^{1} \cup A_{04}^{1}\right)=0$. We show the measure estimate of the most complex condition $A_{k 3}^{1}$. The others can be estimated by the similar method.

Lemma 4.1 (Lemma $1.1 \mathrm{in}$ [23]) Suppose that $f(x)$ is an mth differentiable function on the closure $\bar{I}$ of $I$, where $I \subset \mathbb{R}$ is an interval. Let $I_{h}=\{x|| f(x) \mid<h\}, h>0$. If for some constant $d>0,\left|f^{(m)}(x)\right| \geq d$ for $\forall x \in I$, then meas $I_{h} \leq c^{\frac{1}{m}}$, where $c=2\left(2+3+\cdots+m+d^{-1}\right)$.

We omit the proof of Lemma 4.1. See [23] for details.

Lemma 4.2 For $\tau>2 \kappa+2 b+5, \operatorname{meas}\left(\bigcup_{k \neq 0} A_{k 3}^{1}\right)=\mathcal{O}\left(\epsilon^{\frac{1}{12}} \varepsilon^{-1}\right)$.

Proof Without loss of generality, we assume $i \geq j$ and $i, j \in \mathbb{Z}_{1}$. Since $G_{n_{k} n_{k}}, G_{n_{k} n_{d} i} \leq C$ hold for any $1 \leq k \leq b$ and any $i \in \mathbb{Z}_{1},|\delta| \leq \varepsilon C_{1} \beta^{2}$ is true, where

$$
\begin{aligned}
\delta= & 12 \varepsilon \sum_{k=1}^{b} G_{n_{k} n_{k} i} \varsigma_{k}^{2}+12 \varepsilon \sum_{k=1}^{b} G_{n_{k} n_{k} j} S_{k}^{2} \\
& +48 \varepsilon \sum_{k, d=1, \ldots, b, k \neq d} G_{n_{k} n_{d} i} \varsigma_{k} \varsigma_{d}+48 \varepsilon \sum_{k, d=1, \ldots, b, k \neq d} G_{n_{k} n_{d} j} \zeta_{k} \varsigma_{d},
\end{aligned}
$$

and $C_{1}$ depends on $g_{0}$ and $b$.

Assume

$$
\varepsilon \beta^{2}<1
$$

So, when $i \geq c|k|$,

$$
\begin{aligned}
\frac{\left|\hat{\Omega}_{i}+\hat{\Omega}_{j}\right|}{1+|i-j|} & =\frac{\left|\varepsilon^{-\frac{1}{3}} \lambda_{i}+\varepsilon^{-\frac{1}{3}} \lambda_{j}+\delta\right|}{1+|i-j|} \geq \frac{\left|\varepsilon^{-\frac{1}{3}} \lambda_{i}+\varepsilon^{-\frac{1}{3}} \lambda_{j}\right|}{1+|i-j|}-\frac{\varepsilon \beta^{2} C_{1}}{1+|i-j|} \\
& \geq \frac{\varepsilon^{-\frac{1}{3}} \lambda_{i}-\varepsilon^{-\frac{1}{3}} \lambda_{j}}{2|i-j|}-\varepsilon \beta^{2} C_{1} \geq \frac{\varepsilon^{-\frac{1}{3}}}{2} c|k|-\varepsilon \beta^{2} C_{1}
\end{aligned}
$$

hold. However,

$$
\frac{|\langle k, \hat{\omega}\rangle|}{1+|i-j|} \leq|k||\hat{\omega}| \leq C_{2}|k|\left(\frac{1}{\varepsilon^{\frac{1}{3}}}+\varepsilon \beta^{2}\right)
$$


is true, where $C_{2}$ depends on $\mathcal{I}$ and $g_{0}$. Hence, from (44) and (45), we have

$$
\begin{aligned}
\frac{\left|\hat{\Omega}_{i}+\hat{\Omega}_{j}+\langle k, \hat{\omega}\rangle\right|}{1+|i-j|} & \geq \frac{\left|\hat{\Omega}_{i}-\hat{\Omega}_{j}\right|}{1+|i-j|}-\frac{|\langle k, \hat{\omega}\rangle|}{1+|i-j|} \\
& \geq \frac{\varepsilon^{-\frac{1}{3}}}{2} c|k|-\varepsilon \beta^{2} C_{1}-\frac{C_{2}|k|}{\varepsilon^{\frac{1}{3}}}-C_{2}|k| \varepsilon \beta^{2} .
\end{aligned}
$$

It follows that, when $c>4 C_{2}+4 C_{1}+2$ and $\varepsilon<1$,

$$
\frac{\left|\hat{\Omega}_{i}+\hat{\Omega}_{j}+\langle k, \hat{\omega}\rangle\right|}{1+|i-j|}>1
$$

When $j \geq c|k|$, since $i \geq j, i \geq c|k|$ also holds and we can get the same result. We only need to deal with the case

$$
\max \{i, j\} \leq c|k| .
$$

\section{Suppose that}

$$
f(\xi):=\langle k, \hat{\omega}\rangle+\hat{\Omega}_{i}+\hat{\Omega}_{j} .
$$

The set $B_{k i j}^{1,11}$ is equivalent to

$$
\left\{\bar{\omega} \in \Pi_{1}, \varsigma \in \Pi_{2}:|f|<\frac{\epsilon^{\frac{1}{6}}(|i-j|+1)}{1+\left(\left|k^{(1)}\right|+\left|k^{(2)}\right|\right)^{\tau}}\right\},
$$

where

$$
f=\left\langle k^{(1)}, \omega(\bar{\omega})\right\rangle+\left\langle k^{(2)}, \varpi(\varsigma)\right\rangle+\hat{\Omega}_{i}(\varsigma)+\hat{\Omega}_{j}(\varsigma),
$$

$k^{(1)}:=\left(k_{11}, k_{12}, \ldots, k_{1 m}\right) \in \mathbb{Z}^{\kappa}$ and $k^{(2)}:=\left(k_{21}, k_{22}, \ldots, k_{2 b}\right) \in \mathbb{Z}^{b}$.

Case 1 . When $k^{(1)} \neq 0$, there exists $t_{0}$ satisfying $\frac{\partial f(\xi)}{\partial \bar{\omega}_{0}}=k_{1 t_{0}} \varepsilon \neq 0$. So, $\left|\frac{\partial f(\xi)}{\partial \bar{\omega}_{0}}\right| \geq \varepsilon$. By using Lemma 4.1, from (46), we have that

$$
\begin{aligned}
\operatorname{meas}\left(\bigcup_{k^{(1)} \neq 0} A_{k 3}^{1}\right) & \leq \sum_{k \neq 0} \sum_{i, j \leq c|k|} \frac{2 \epsilon^{\frac{1}{6}}(|i-j|+1)}{\varepsilon A_{k}} \leq \sum_{k \neq 0} \frac{2 \epsilon^{\frac{1}{6}} 3 c|k|}{\varepsilon|k|^{\tau}} \\
& \leq \sum_{k \neq 0} \frac{6 c \epsilon^{\frac{1}{6}}}{\varepsilon|k|^{\tau-1}} \leq \sum_{l \neq 0} \frac{6 c \epsilon^{\frac{1}{6}}}{\varepsilon l^{\tau-1}} 2^{\kappa+b} l^{\kappa+b-1} \leq C \frac{\epsilon^{\frac{1}{6}}}{\varepsilon} \sum_{l \neq 0} \frac{1}{l^{\tau-(\kappa+b)}} .
\end{aligned}
$$

When $\tau>(\kappa+b)+1$, the last series $\sum_{l \neq 0} l^{\tau-(\kappa+b)}$ is convergent. Therefore, meas $\left(\bigcup_{k \neq 0} A_{k 3}^{1}\right)=$ $\mathcal{O}\left(\epsilon^{\frac{1}{6}} \varepsilon^{-1}\right)$.

Case 2. When $k^{(1)}=0$ and $k^{(2)} \neq 0$ hold, we have $f(\xi)=\left\langle k^{(2)}, \varpi\right\rangle+\hat{\Omega}_{i}+\hat{\Omega}_{j}$. Clearly, the 1 st to $\kappa$ th elements of the vector $\frac{\partial f(\xi)}{\partial \xi}$ are zeros. We only calculate the $(\kappa+1)$ th to $(\kappa+b)$ th 
elements of the vector $\frac{\partial^{2} f(\xi)}{\partial \xi^{2}}$. It follows that

$$
\begin{aligned}
& \frac{\pi^{2}}{2 \varepsilon g_{0}} \cdot \frac{\partial^{2} f}{\partial \varsigma_{1}^{2}}=10 k_{21}+18 k_{22}+\cdots+18 k_{2 b}+3\left(c_{1}+c_{1}^{\prime}\right), \\
& \frac{\pi^{2}}{2 \varepsilon g_{0}} \cdot \frac{\partial^{2} f}{\partial \zeta_{2}^{2}}=18 k_{21}+10 k_{22}+\cdots+18 k_{2 b}+3\left(c_{2}+c_{2}^{\prime}\right), \\
& \cdots \\
& \frac{\pi^{2}}{2 \varepsilon g_{0}} \cdot \frac{\partial^{2} f}{\partial \varsigma_{b}^{2}}=18 k_{21}+18 k_{22}+\cdots+10 k_{2 b}+3\left(c_{b}+c_{b}^{\prime}\right),
\end{aligned}
$$

where $c_{i}, c_{i}^{\prime}=5$ or 6 for $i=1, \ldots, b$, and no more than two $5 \mathrm{~s}$ in $\left\{c_{1}, c_{2}, \ldots, c_{b}, c_{1}^{\prime}, c_{2}^{\prime}, \cdots, c_{b}^{\prime}\right\}$. Then there exists $t_{0}\left(1 \leq t_{0} \leq b\right)$ s.t.

$$
\frac{\pi^{2}}{2 \varepsilon g_{0}} \cdot \frac{\partial^{2} f}{\partial \zeta_{t_{0}}^{2}} \neq 0
$$

Otherwise, suppose that the equalities in (47) are all zeros and we can get that

$$
k_{21}=\frac{-3}{8(9 b-4)}\left[(13-9 b)\left(c_{1}+c_{1}^{\prime}\right)+9\left(c_{2}+c_{2}^{\prime}+\cdots+c_{b}+c_{b}^{\prime}\right)\right] .
$$

However, contradictions follow according to the cases below.

Case a. Two " $5 \mathrm{~s}$ " in $\left\{c_{1}, c_{2}, \ldots, c_{b}, c_{1}^{\prime}, c_{2}^{\prime}, \ldots, c_{b}^{\prime}\right\}$.

Case a.1. Assume $c_{1}=5, c_{1}^{\prime}=6$ and there is one 5 in $\left\{c_{2}, \ldots, c_{b}, c_{2}^{\prime}, \ldots, c_{b}^{\prime}\right\}$. It follows that $k_{21}=-\frac{3(9 b+26)}{72 b-32}$. Obviously, $k_{21} \notin \mathbb{Z}$. Similarly, we get the same result for the case that $c_{1}=6$, $c_{1}^{\prime}=5$.

Case a.2. Assume $c_{1}=6, c_{1}^{\prime}=6$ and there are two $5 \mathrm{~s}$ in $\left\{c_{2}, \ldots, c_{b}, c_{2}^{\prime}, \ldots, c_{b}^{\prime}\right\}$. It follows that $\left|k_{21}\right|=-\frac{45}{4(9 b-4)}$. So $k_{21} \notin \mathbb{Z}$.

Case a.3. Assume $c_{1}=5$ and $c_{1}^{\prime}=5$. It follows that $\left|k_{21}\right|=\frac{3(18 b+22)}{8(9 b-4)}$. If $b=2, \ldots, 5$, it is clear that $k_{21} \notin \mathbb{Z}$. If $b \geq 6$, then $0<\left|k_{21}\right|<1$.

Case b. Only one " 5 " in $\left\{c_{1}, c_{2}, \ldots, c_{b}, c_{1}^{\prime}, c_{2}^{\prime}, \ldots, c_{b}^{\prime}\right\}$.

Case b.1. Assume $c_{1}=5$ or $c_{1}^{\prime}=5$. It follows that $\left|k_{21}\right|=\frac{105+27 b}{72 b-32}$. If $b=2,\left|k_{21}\right|=\frac{159}{112}$. If $b=2,\left|k_{21}\right|=\frac{93}{92}$. If $b \geq 4$, then $0<\left|k_{21}\right|<1$.

Case b. 2 Assume $c_{i_{0}}=5$ or $c_{i_{0}}^{\prime}=5$ for $i_{0} \neq 1$. It follows that $\left|k_{21}\right|=\frac{117}{8(9 b-4)}$. So $k_{21} \notin \mathbb{Z}$.

Case c. No " 5 " in $\left\{c_{1}, c_{2}, \ldots, c_{b}, c_{1}^{\prime}, c_{2}^{\prime}, \ldots, c_{b}^{\prime}\right\}$. It follows that $k_{21}=-\frac{18}{9 b-4}$. If $b=2$, then $k_{21}=-\frac{9}{7}$. If $b \geq 3$, then $0<\left|k_{21}\right|<1$.

Since $k_{21} \in \mathbb{Z}$, we get contradictions. Thus there exists $t_{0}\left(1 \leq t_{0} \leq b\right)$ s.t. (48) holds. Furthermore, we can have $\left|\frac{\partial^{2} f}{\partial s_{t_{0}}^{2}}\right| \geq \frac{2 g_{0}}{\pi^{2}} \varepsilon$.

It follows that, by using of Lemma 4.1,

$$
\begin{aligned}
\operatorname{meas}\left(\bigcup_{k^{(1)}=0, k^{(2)} \neq 0} A_{k 3}^{1}\right) & \leq \operatorname{meas}\left(\bigcup_{k \neq 0} \bigcup_{i, j \leq c|k|} B_{k i j}^{1,11}\right) \\
& \leq \sum_{k \neq 0} \sum_{i, j \leq c|k|} C\left(\frac{\epsilon^{\frac{1}{6}}|k|}{1+|k|^{\tau}}\right)^{\frac{1}{2}} \cdot \frac{|k|^{2}}{\varepsilon} \\
& \leq C \epsilon^{\frac{1}{12}} \varepsilon^{-1} \sum_{k \neq 0} \frac{|k|^{2}}{|k|^{\frac{\tau-1}{2}}}
\end{aligned}
$$




$$
\begin{aligned}
& \leq C \epsilon^{\frac{1}{12}} \varepsilon^{-1} \sum_{l \neq 0} \frac{1}{l^{\frac{\tau}{2}-\frac{3}{2}-b-\kappa}} \\
& =\mathcal{O}\left(\epsilon^{\frac{1}{12}} \varepsilon^{-1}\right) .
\end{aligned}
$$

Therefore, when $\tau>2 \kappa+2 b+5, \operatorname{meas}\left(\bigcup_{k \neq 0} A_{k 3}^{1}\right)=\mathcal{O}\left(\epsilon^{\frac{1}{6}} \varepsilon^{-1}\right)+\mathcal{O}\left(\epsilon^{\frac{1}{12}} \varepsilon^{-1}\right)$. This completes the proof.

From (42), meas $\left(\bigcup_{k \neq 0} A_{k 3}^{1}\right)=\mathcal{O}\left(\varepsilon^{-\frac{15}{16}}\right)$ holds.

Similarly, we prove the same results for $A_{k 1}^{1}, A_{k 2}^{1}$, and $A_{k 4}^{1}$. Furthermore, the following lemma holds.

Lemma 4.3 For $\tau>2 \kappa+2 b+5$, meas $\left(\left(\bigcup_{k \neq 0} A_{k 1}^{1}\right) \cup\left(\bigcup_{k}\left(A_{k 2}^{1} \cup A_{k 3}^{1} \cup A_{k 4}^{1}\right)\right)\right)=\mathcal{O}\left(\epsilon^{\frac{1}{12}} \varepsilon^{-1}\right)=$ $\mathcal{O}\left(\varepsilon^{-\frac{15}{16}}\right)$ is true.

\section{Conclusions}

In this work, we proved that there exists a positive measure Cantor manifold of real analytic rotational $\kappa+b$-tori for equation (1). All the tori are small amplitude, linearly stable, and all their orbits have zero Lyapunov exponents.

Equation (1) has three characters. It is quasi-periodically forced, the nonlinearity is $x$ dependent, and the nonlinearity is quintic. To our best knowledge, it is the first time to consider the existence of quasi-periodic solutions for (1) by the KAM method. We estimate measures of infinitely many small divisors, build a variable-coefficient symplectic transformation, and show an infinite-dimensional KAM theorem for non-autonomous Hamiltonian systems.

\section{Appendix}

Proof of Lemma 2.3 Let $q \in l^{a, s}$. Considered as a function on $[-\pi, \pi], u(t, x)=\sum_{j \geq 1} q_{j}(t) \times$ $\phi_{j}(x)$ is in $W^{a, s}$, with

$$
\|u\|_{a, s}=\|q\|_{a, s} .
$$

For every $\vartheta \in D_{1}\left(\sigma_{1}\right)$, we expand $g$ in a Fourier series

$$
g(\vartheta, x)=\frac{1}{\sqrt{2 \pi}} \sum_{j} g_{j}(\vartheta) e^{\mathrm{i} j x} .
$$

Using Lemma A.1 in [22], for every $j \in \mathbb{Z}$, we have that

$$
\left|g_{j}(\vartheta)\right| \leq|g|_{2 a} e^{-2 a|j|}
$$

holds. So

$$
\begin{aligned}
\left(\|g\|_{a, s}\right)^{2} & =\left|g_{0}(\vartheta)\right|^{2}+\sum_{j \neq 0}\left|g_{j}(\vartheta)\right|^{2}|j|^{2 s} e^{2 a|j|} \\
& <\sum|g|_{2 a}^{2} e^{-4 a|j|}[j]^{2 s} e^{2 a|j|}
\end{aligned}
$$




$$
\begin{aligned}
& <|g|_{\sigma_{1}, 2 a}^{2} \sum_{j} e^{-2 a|j|}[j]^{2 s} \\
& <C|g|_{\sigma_{1}, 2 a}^{2}
\end{aligned}
$$

where $[j]:=\max \{|j|, 1\}$. Therefore, $g \in W^{a, s}[-\pi, \pi]$. It follows from (50) that, by Lemma 2.2 and the analyticity of $g$ and $|u|^{4} u$, the function $g(\vartheta, x)|u|^{4} u$ also belongs to $W^{a, s}[-\pi, \pi]$ with

$$
\left\|g(\vartheta, x)|u|^{4} u\right\|_{a, s} \leq C|g|_{\sigma_{1}, 2 a}\left(\|q\|_{a, s}\right)^{5}
$$

in a neighborhood of the origin, where $C$ depends on $s$ and $a$. On the other hand, since

$$
\frac{\partial G}{\partial \bar{q}_{j}}=\int_{0}^{\pi} g(\vartheta, x)|u|^{4} u \phi_{j}(x) d x
$$

the components of $G_{\bar{q}}$ are the Fourier coefficients of $g(\vartheta, x)|u|^{4} u$, so $G_{\bar{q}}$ belongs to $l^{a, s}$ with

$$
\left\|G_{\bar{q}}\right\|_{a, s} \leq C\left\|g(\vartheta, x)|u|^{4} u\right\|_{a, s} \leq C|g|_{\sigma_{1}, 2 a}\left(\|q\|_{a, s}\right)^{5}
$$

where the last $C$ depends on $s$ and $a$. The regularity of $G_{\bar{q}}$ follows from the regularity of its components and its local boundedness.

Proof of Lemma 3.1 Let $0 \neq k \in \mathbb{Z}^{\kappa}$,

$$
\mathcal{R}_{k}^{1}=\left\{\omega \in[\varrho, 2 \varrho]^{\kappa}:|\langle k, \omega\rangle|<\frac{\varrho \varepsilon^{\eta_{0}}}{|k|^{\kappa+\eta_{1}}}\right\} .
$$

Consider two hyperplanes $\langle k, \omega\rangle= \pm \frac{\varrho \varepsilon^{\eta_{0}}}{|k|^{k+\eta_{1}}}$. We have

$$
\text { meas } \mathcal{R}_{k}^{1} \leq \kappa|k|^{-1}(\sqrt{2} \varrho)^{\kappa-1} \frac{2 \varrho \varepsilon^{\eta_{0}}}{|k|^{\kappa+\eta_{1}}} \leq \frac{2(\sqrt{2})^{\kappa-1} \kappa \varepsilon^{\eta_{0}}}{|k|^{\kappa+\eta_{1}+1}} \varrho^{\kappa} .
$$

It follows that

$$
\text { meas } \bigcup_{0 \neq k \in \mathbb{Z}^{\kappa}} \mathcal{R}_{k}^{1} \leq \sum_{1 \leq k \in \mathbb{Z}^{\kappa}} \text { meas } \mathcal{R}_{k}^{1} \leq 2(\sqrt{2})^{\kappa-1} \kappa \varepsilon^{\eta_{0}} \varrho^{\kappa} \sum_{1 \leq k \in \mathbb{Z}^{\kappa}} \frac{1}{|k|^{\kappa+\eta_{1}+1}} \text {. }
$$

So, from (30), when $\eta_{1}>-1$,

$$
\text { meas } \bigcup_{0 \neq k \in \mathbb{Z}^{\kappa}} \mathcal{R}_{k}^{1} \leq C_{1} \varepsilon^{\eta_{0}} \varrho^{\kappa} \sum_{l=1}^{\infty} 2^{\kappa} l^{\kappa-1} l^{-\left(\kappa+\eta_{1}+1\right)} \leq C_{1} \varepsilon^{\eta_{0}} \varrho^{\kappa} \text {. }
$$

Therefore this lemma is true when we assume that $\underline{\Omega}=[\varrho, 2 \varrho]^{\kappa} \backslash\left(\bigcup_{0 \neq k \in \mathbb{Z}^{\kappa}} \mathcal{R}_{k}^{1}\right)$.

Proof of Lemma 3.2 Clearly,

$$
\langle k, \omega\rangle+\lambda_{i}+\lambda_{j}+\lambda_{d}-\lambda_{l}-\lambda_{m}-\lambda_{n}=\langle k, \omega\rangle+i^{2}+j^{2}+d^{2}-l^{2}-m^{2}-n^{2} .
$$


Assume that

$$
\begin{aligned}
& f_{\vec{\zeta}, k}=\langle k, \omega\rangle+i^{2}+j^{2}+d^{2}-l^{2}-m^{2}-n^{2}, \\
& \mathcal{R}_{\vec{\zeta}, k}=\left\{\omega \in[\varrho, 2 \varrho]^{\kappa}:\left|f_{\vec{\zeta}, k}\right|<\frac{\varrho \varepsilon^{\eta_{0}}}{|k|^{\kappa+\eta_{1}}}\right\}, \\
& \tilde{\Omega}=\bigcup_{|k| \geq 1} \bigcup_{\vec{\zeta} \in \bar{\Delta}} \mathcal{R}_{\vec{\zeta}, k}, \quad \Omega^{0}=\bigcup_{|k| \geq 1} \bigcup_{\vec{\zeta} \in \Delta_{0}} \mathcal{R}_{\vec{\zeta}, k}, \\
& \Omega^{1}=\bigcup_{|k| \geq 1} \bigcup_{\vec{\zeta} \in \Delta_{1}} \mathcal{R}_{\vec{\zeta}, k}, \quad \text { and } \quad \Omega^{2}=\bigcup_{|k| \geq 1} \bigcup_{\vec{\zeta} \in \Delta_{2}} \mathcal{R}_{\vec{\zeta}, k} .
\end{aligned}
$$

It is evident that $\tilde{\Omega}=\Omega^{0} \cup \Omega^{1} \cup \Omega^{2}$. Similar to (52), we can attain meas $\mathcal{R}_{\vec{\zeta}, k} \leq C \frac{\varepsilon^{\eta_{0}} \varrho^{\kappa}}{|k|^{k+\eta} 1^{+1}}$, where $C$ depends on $\kappa$.

Case 1. If $\vec{\zeta} \in \Delta_{0}$, then from (30)

$$
\text { meas } \begin{aligned}
\Omega^{0} & \leq C \sum_{|k| \geq 1} \frac{\varepsilon^{\eta_{0}} \varrho^{\kappa}}{|k|^{\kappa+\eta_{1}+1}}\left(n_{b}-n_{1}+1\right)^{6} \\
& \leq C\left(n_{b}-n_{1}+1\right)^{6} \varrho^{\kappa} \varepsilon^{\eta_{0}} \sum_{1 \leq|k|=l} \frac{1}{l^{\kappa+\eta_{1}+1}} 2^{\kappa} l^{\kappa-1} .
\end{aligned}
$$

If $\eta_{1}>-1$, then $\sum_{l \geq 1} \frac{1}{l_{1}+2}$ is convergent and meas $\Omega^{0} \leq C \varrho^{\kappa} \varepsilon^{\eta_{0}}$, where the constant $C$ depends on $n_{1}, n_{b}$, and $\kappa$.

Case 2. If $\vec{\varsigma} \in \Delta_{1}$, we assume that $l \notin \mathcal{I}$ without loss of generality. So,

$$
\left|\langle k, \omega\rangle+i^{2}+j^{2}+d^{2}-m^{2}-n^{2}\right| \leq 2 \varrho|k|+5 n_{b}^{2}
$$

When $l>\sqrt{2 \varrho|k|+5 n_{b}^{2}+1}$ and $\varepsilon$ is small enough,

$$
\begin{aligned}
\left|f_{\vec{s}, k}\right| & \geq l^{2}-\left|\langle k, \omega\rangle+i^{2}+j^{2}+d^{2}-m^{2}-n^{2}\right| \\
& >2 \varrho|k|+5 n_{b}^{2}+1-\left(2 \varrho|k|+5 n_{b}^{2}\right)=1>\frac{\varepsilon^{\eta_{0}} \varrho^{\kappa}}{|k|^{\kappa+\eta_{1}}},
\end{aligned}
$$

which implies that one only needs to consider the case $1 \leq l \leq \sqrt{2 \varrho|k|+5 n_{b}^{2}+1}$. Therefore, if $\eta_{1}>0$,

$$
\begin{aligned}
\operatorname{meas} \Omega^{1} & \leq C \sum_{|k| \geq 1} \frac{\varepsilon^{\eta_{0}} \varrho^{\kappa}}{|k|^{\kappa+\eta_{1}+1}}\left(n_{b}-n_{1}+1\right)^{5} \sqrt{2 \varrho|k|+5 n_{b}^{2}+1} \\
& \leq C \varrho^{\kappa} \varepsilon^{\eta_{0}}\left(n_{b}-n_{1}+1\right)^{5} \sum_{|k| \geq 1} \frac{1}{|k|^{\kappa+\eta_{1}}} \\
& \leq C \varrho^{\kappa} \varepsilon^{\eta_{0}}\left(n_{b}-n_{1}+1\right)^{5} \sum_{l \geq 1} 2^{\kappa} l^{\kappa-1} \frac{1}{l^{\kappa+\eta_{1}}} \leq C \varrho^{\kappa} \varepsilon^{\eta_{0}}
\end{aligned}
$$

where the constant $C$ depends on $n_{1}, n_{b}, \varrho$, and $\kappa$.

Case 3. If $\vec{\zeta} \in \Delta_{2}$, we discuss the cases $d, l \notin \mathcal{I}$ and $i, d \notin \mathcal{I}$. Other cases can be proved in the same way. 
Case 3.1. Assume $d, l \notin \mathcal{I}$. We divide this case into two cases: 3.1.1 and 3.1.2 below.

Case 3.1.1. If $d=l$, then $\left|f_{\vec{s}, k}\right|=\left|\langle k, \omega\rangle+i^{2}+j^{2}-m^{2}-n^{2}\right|$. Supposing that

$$
\Omega^{2,1}=\bigcup_{|k| \geq 1} \bigcup_{\vec{\zeta} \in \Delta_{2}, d=l \notin \mathcal{I}, i, j, m, n \in \mathcal{I}} \mathcal{R}_{\vec{\zeta}, k}
$$

then we have, if $\eta_{1}>-1$,

$$
\begin{aligned}
\operatorname{meas} \Omega^{2,1} & \leq C \sum_{|k| \geq 1} \frac{\varrho^{\kappa} \varepsilon^{\eta_{0}}}{|k|^{\kappa+\eta_{1}+1}}\left(n_{b}-n_{1}+1\right)^{4} \\
& \leq C \varrho^{\kappa} \varepsilon^{\eta_{0}}\left(n_{b}-n_{1}+1\right)^{4} \sum_{|k| \geq 1} \frac{1}{|k|^{\kappa+\eta_{1}+1}} \\
& \leq C \varrho^{\kappa} \varepsilon^{\eta_{0}}\left(n_{b}-n_{1}+1\right)^{4} \sum_{l \geq 1} 2^{\kappa} l^{\kappa-1} \frac{1}{l^{\kappa+\eta_{1}+1}} \leq C \varrho^{\kappa} \varepsilon^{\eta_{0}}
\end{aligned}
$$

where the constant $C$ depends on $n_{1}, n_{b}$, and $\kappa$.

Case 3.1.2. If $d \neq l$, without loss of generality, we can suppose $d>l$ or $d-l:=p \geq 1$. Clearly,

$$
d^{2}-l^{2}=(d-l)(d+l)=p(2 l+p) .
$$

If $l>\tilde{N}:=\varrho|k|+2 n_{b}^{2}$, it is derived that $\left|d^{2}-l^{2}\right| \geq 2 l+p>2 \tilde{N}+1$. Since

$$
\left|\langle k, \omega\rangle+i^{2}+j^{2}-m^{2}-n^{2}\right| \leq 2 \varrho|k|+4 n_{b}^{2}
$$

and

$$
\left|f_{\vec{\zeta}, k}\right| \geq\left|d^{2}-l^{2}\right|-\left|\langle k, \omega\rangle+i^{2}+j^{2}-m^{2}-n^{2}\right|
$$

we have

$$
\left|f_{\vec{\zeta}, k}\right|>2 \tilde{N}+1-2 \varrho|k|-4 n_{b}^{2}=1>\frac{\varrho \varepsilon^{\eta_{0}}}{|k|^{\kappa+\eta_{1}}}
$$

holds as $\varepsilon$ is small enough. Therefore, we only need to consider the case $1 \leq l \leq \tilde{N}$. If $p>\tilde{N}+1$, (53) induces that

$$
\left|d^{2}-l^{2}\right|=2 p l+p^{2}>2 p=2 \tilde{N}+2
$$

It follows from (54) and (55) that

$$
\begin{aligned}
\left|f_{\vec{\zeta}, k}\right| & \geq 2 \tilde{N}+2-2 \varrho|k|-4 n_{b}^{2} \\
& =2 \varrho|k|+4 n_{b}^{2}+2-2 \varrho|k|-4 n_{b}^{2}=2>\frac{\varrho^{\kappa} \varepsilon^{\eta_{0}}}{|k|^{\kappa+\eta_{1}}}
\end{aligned}
$$


as $\varepsilon$ is small enough. So, we only need to consider the case $1 \leq l \leq \tilde{N}$ and $1 \leq p \leq \tilde{N}+1$. In this case, $1 \leq d=l+p \leq 2 \tilde{N}+1$. Supposing that

$$
\Omega^{2,2}=\bigcup_{|k| \geq 1} \bigcup_{\vec{\zeta} \in \Delta_{2}, d \neq l, d, l \notin \mathcal{I}, i, j, m, n \in \mathcal{I}} \mathcal{R}_{\vec{\zeta}, k}
$$

then we have, if $\eta_{1}>1$,

$$
\begin{aligned}
\operatorname{meas} \Omega^{2,2} & \leq C \sum_{|k| \geq 1} \frac{\varepsilon^{\eta_{0}} \varrho^{\kappa}}{|k|^{\kappa+\eta_{1}+1}}\left(n_{b}-n_{1}+1\right)^{4}\left(\varrho|k|+2 n_{b}^{2}\right)\left(2 \varrho|k|+4 n_{b}^{2}+1\right) \\
& \leq C \varrho^{\kappa} \varepsilon^{\eta_{0}}\left(n_{b}-n_{1}+1\right)^{4} \sum_{|k| \geq 1} \frac{1}{|k|^{\kappa+\eta_{1}-1}} \\
& \leq C \varrho^{\kappa} \varepsilon^{\eta_{0}}\left(n_{b}-n_{1}+1\right)^{4} \sum_{l \geq 1} 2^{\kappa} l^{\kappa-1} \frac{1}{l^{\kappa+\eta_{1}-1}} \leq C \varrho^{\kappa} \varepsilon^{\eta_{0}}
\end{aligned}
$$

where the constant $C$ depends on $\varrho, n_{1}, n_{b}$, and $\kappa$.

Case 3.2. Assume $i, d \notin \mathcal{I}$. When $\max \{i, d\}>\sqrt{2 \varrho|k|+4 n_{b}^{2}+1}$, we have that

$$
\begin{aligned}
\left|f_{\vec{\zeta}, k}\right| & \geq\left|i^{2}+d^{2}\right|-\left|\langle k, \omega\rangle+j^{2}-l^{2}-m^{2}-n^{2}\right| \\
& \geq\left(2 \varrho|k|+4 n_{b}^{2}+1\right)-\left(2 \varrho|k|+4 n_{b}^{2}\right)=1 .
\end{aligned}
$$

So, we only need to consider the case $1 \leq i, d \leq \sqrt{2 \varrho|k|+4 n_{b}^{2}+1}$. Suppose that

$$
\Omega^{2,3}=\bigcup_{|k| \geq 1} \bigcup_{\vec{\zeta} \in \Delta_{2}, i, d \notin \mathcal{I}, j, l, m, n \in \mathcal{I}} \mathcal{R}_{\vec{\zeta}, k}
$$

then we have, if $\eta_{1}>0$,

$$
\begin{aligned}
\text { meas } \Omega^{2,3} & \leq C \sum_{|k| \geq 1} \frac{\varrho^{\kappa} \varepsilon^{\eta_{0}}}{|k|^{\kappa+\eta_{1}+1}}\left(n_{b}-n_{1}+1\right)^{4}\left(2 \varrho|k|+4 n_{b}^{2}+1\right) \\
& \leq C \varrho^{\kappa} \varepsilon^{\eta_{0}}\left(n_{b}-n_{1}+1\right)^{4} \sum_{|k| \geq 1} \frac{1}{|k|^{\kappa+\eta_{1}}} \\
& \leq C \varrho^{\kappa} \varepsilon^{\eta_{0}}\left(n_{b}-n_{1}+1\right)^{4} \sum_{l \geq 1} 2^{\kappa} l^{\kappa-1} \frac{1}{l^{\kappa+\eta_{1}}} \leq C \varrho^{\kappa} \varepsilon^{\eta_{0}},
\end{aligned}
$$

where the constant $C$ depends on $\varrho, n_{1}, n_{b}$, and $\kappa$.

Thus, if $\eta_{1}>1$, for $\vec{\zeta} \in \Delta_{2}$, there is a constant $C$ satisfying that meas $\Omega^{2} \leq C \varrho^{\kappa} \varepsilon^{\eta_{0}}$. Overall, when $\eta_{1}>1$,

$$
\text { meas } \bar{\Omega} \geq\left(1-C_{2} \varepsilon^{\eta_{0}}\right) \varrho^{\kappa},
$$

where we suppose that $\bar{\Omega}=[\varrho, 2 \varrho]^{\kappa} \backslash \tilde{\Omega}$. The constant $C_{2}$ depends on $\varrho, n_{1}, n_{b}$, and $\kappa$. The proof is completed. 
Proof of Lemma 3.3 By the Schwarz inequality and from (30),

$$
\begin{aligned}
\left|\sum_{k} x_{k}\right|^{2} & =\left|\sum_{k} \frac{[k]^{\kappa+\frac{1}{2}} x_{k}}{[k]^{\kappa+\frac{1}{2}}}\right|^{2} \leq \sum_{k} \frac{1}{[k]^{2 \kappa+1}} \sum_{k}[k]^{2 \kappa+1}\left|x_{k}\right|^{2} \\
& =\left(\sum_{k=0} \frac{1}{[k]^{2 \kappa+1}}+\sum_{k \neq 0} \frac{1}{[k]^{2 \kappa+1}}\right) \sum_{k}[k]^{2 \kappa+1}\left|x_{k}\right|^{2} \\
& =\left(1+\sum_{|k|=1} \frac{1}{[k]^{2 \kappa+1}}\right) \sum_{k}[k]^{2 \kappa+1}\left|x_{k}\right|^{2} \\
& \leq\left(1+\sum_{a=1}^{\infty} \frac{1}{a^{2 \kappa+1}} 2^{\kappa} a^{\kappa-1}\right) \sum_{k}[k]^{2 \kappa+1}\left|x_{k}\right|^{2} \\
& =\left(1+2^{\kappa} \sum_{a=1}^{\infty} \frac{1}{a^{\kappa+2}}\right) \sum_{k}[k]^{2 \kappa+1}\left|x_{k}\right|^{2},
\end{aligned}
$$

where $\kappa>0$. Since the series $\sum_{a=1}^{\infty} \frac{1}{a^{\kappa+2}}$ is convergent,

$$
\left|\sum_{k} x_{k}\right|^{2} \leq c \sum_{k}[k]^{2 \kappa+1}\left|x_{k}\right|^{2} \text {. }
$$

This completes the proof.

\section{Acknowledgements}

We would like to thank the referees for their valuable comments and suggestions to improve our paper.

\section{Funding}

This work is partially supported by the National Natural Science Foundation of China (Grant Nos. 11601270, 11626087, and 11701567). This work is also partially supported by the Research Foundation for Doctor Programme of Henan Polytechnic University (Grant B2016-58).

\section{Availability of data and materials}

Data sharing not applicable to this article as no data sets were generated or analyzed during the current study.

\section{Competing interests}

The authors declare that there is no conflict of interests regarding the publication of this article.

\section{Authors' contributions}

YW, JL, and MZ all contributed to this study. YW proved the results and drafted the article. YW, JL, and MZ reviewed and edited the manuscript. All the authors have read and approved the final manuscript.

\section{Author details}

${ }^{1}$ School of Mathematics and Quantitative Economics, Shandong University of Finance and Economics, Jinan, P.R. China. ${ }^{2}$ School of Mathematics and Information Science, Henan Polytechnic University, Jiaozuo, P.R. China. ${ }^{3}$ College of Science, China University of Petroleum, Qingdao, P.R. China.

\section{Publisher's Note}

Springer Nature remains neutral with regard to jurisdictional claims in published maps and institutional affiliations.

Received: 13 December 2017 Accepted: 4 May 2018 Published online: 11 May 2018

\section{References}

1. Kuksin, S.B.: Hamiltonian perturbations of infinite-dimensional linear systems with imaginary spectrum. Funkc. Anal. Prilozh. 21, 22-37 (1987)

2. Kuksin, S.B.: Perturbations of quasiperiodic solutions of infinite-dimensional Hamiltonian systems. Izv. Akad. Nauk SSSR, Ser. Mat. 52, 41-63 (1988)

3. Kuksin, S.B.: Nearly Integrable Infinite-Dimensional Hamiltonian Systems. Lecture Notes in Math., vol. 1556. Springer, New York (1993) 
4. Wayne, C.: Periodic and quasi-periodic solutions of nonlinear wave equations via KAM theory. Commun. Math. Phys. $127,479-528(1990)$

5. Kuksin, S.B., Pöschel, J.: Invariant Cantor manifolds of quasiperiodic oscillations for a nonlinear Schrödinger equation. Ann. Math. 143, 149-179 (1996)

6. Geng, J.S., You, J.G.: A KAM theorem for one-dimensional Schrödinger equation with periodic boundary conditions. J. Differ. Equ. 209, 1-56 (2005)

7. Geng, J.S., You, J.G.: A KAM theorem for Hamiltonian partial differential equations in higher dimensional spaces. Commun. Math. Phys. 262, 343-372 (2006)

8. Liu, J.J., Yuan, X.P.: KAM for the derivative nonlinear Schrödinger equation with periodic boundary conditions. J. Differ. Equ. 256, 1627-1652 (2014)

9. Liang, Z.G., You, J.G.: Quasi-periodic slutions for 1D Schrödinger equations with higher order nonlinearity. SIAM J. Math. 36, 1965-1990 (2005)

10. Geng, J.S., Yi, Y.F.: Quasi-periodic slutions in a nonlinear Schrödinger equation. J. Differ. Equ. 233, 512-542 (2007)

11. Liang, Z.G.: Quasi-periodic solutions for 1D Schrödinger equation with the nonlinearity $|u|^{2 p} u$. J. Differ. Equ. 244 2185-2225 (2008)

12. Geng, J.S., Wu, J.: Real analytic quasi-periodic solutions for the derivative nonlinear Schrödinger equations. J. Math Phys. 53, $102702(2012)$

13. Gao, M.N., Liu, J.J.: Quasi-periodic solutions for derivative nonlinear derivative nonlinear Schrödinger equation. Discrete Contin. Dyn. Syst. 32, 2101-2123 (2012)

14. Eliasson, L.H., Kuksin, S.B.: KAM for the non-linear Schrödinger equation. Ann. Math. 172, 371-435 (2010)

15. Bambusi, D., Berti, M., Magistrelli, E.: Degenerate KAM theory for partial differential equations. J. Differ. Equ. 250, 3379-3397 (2011)

16. Eliasson, L.H., Grébert, B., Kuksin, S.B.: KAM for the nonlinear beam equation. Geom. Funct. Anal. 26, 1588-1715 (2016)

17. Jiao, L., Wang, Y.Q.: The construction of quasi-periodic solutions of quasi-periodic forced Schrödinger equation. Commun. Pure Appl. Anal. 8, 1585-1606 (2009)

18. Berti, M., Bolle, P.: Quasi-periodic solutions of nonlinear Schrodinger equations on $\mathbb{T}^{d}$. Rend. Lincei Mat. Appl. 22, 223-236 (2011)

19. Berti, M., Biasco, L., Procesi, M.: Existence and stability of quasi-periodic solutions for derivative wave equations. Rend. Lincei Mat. Appl. 24, 199-214 (2013)

20. Si, J.G.: Quasi-periodic solutions of a non-autonomous wave equations with quasi-periodic forcing. J. Differ. Equ. 252, 5274-5360 (2012)

21. Baldi, P., Berti, M., Montalto, R.: KAM for quasi-linear and fully nonlinear forced KdV. Mathematics 24, 437-450 (2012)

22. Pöschel, J.: A lecture on the classical KAM theorem. Proc. Symp. Pure Math. 69, 707-732 (2001)

23. Xu, J.X., Qiu, Q.J., You, J.G.: A KAM therorem of degenerate infinite dimensional Hamiltonian systems (II). Sci. China Ser. A 39, 384-394 (1996)

\section{Submit your manuscript to a SpringerOpen ${ }^{\circ}$ journal and benefit from:}

- Convenient online submission

- Rigorous peer review

Open access: articles freely available online

- High visibility within the field

- Retaining the copyright to your article

Submit your next manuscript at $\gg$ springeropen.com 